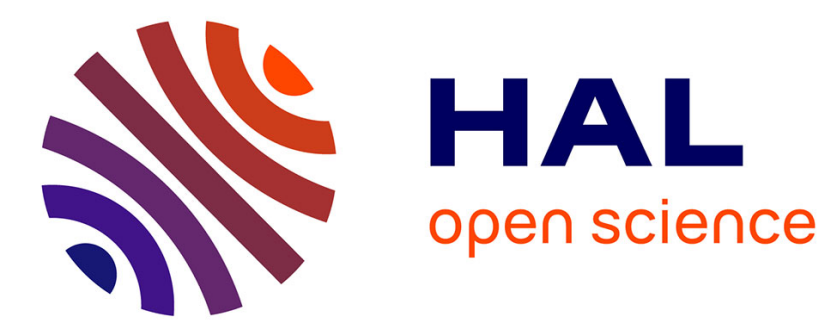

\title{
Air Traffic Assignment for Intensive Urban Air Mobility Operations
}

\author{
Zhengyi Wang, Daniel Delahaye, Jean-Loup Farges, Sameer Alam
}

\section{To cite this version:}

Zhengyi Wang, Daniel Delahaye, Jean-Loup Farges, Sameer Alam. Air Traffic Assignment for Intensive Urban Air Mobility Operations. Journal of Aerospace Information Systems, 2021. hal-03082598

\section{HAL Id: hal-03082598 \\ https://hal.science/hal-03082598}

Submitted on 8 Nov 2021

HAL is a multi-disciplinary open access archive for the deposit and dissemination of scientific research documents, whether they are published or not. The documents may come from teaching and research institutions in France or abroad, or from public or private research centers.
L'archive ouverte pluridisciplinaire HAL, est destinée au dépôt et à la diffusion de documents scientifiques de niveau recherche, publiés ou non, émanant des établissements d'enseignement et de recherche français ou étrangers, des laboratoires publics ou privés. 


\title{
Air Traffic Assignment for Intensive Urban Air Mobility Operations
}

\author{
Zhengyi Wang * and Daniel Delahaye ${ }^{\dagger}$ \\ OPTIM Group, ENAC Lab, French Civil Aviation University, Toulouse 31055, France \\ Jean-Loup Farges \\ ONERA, Toulouse 31000, France \\ Sameer Alam ${ }^{\S}$ \\ ATMRI, Nanyang Technological University, Singapore 639798
}

In high-density Urban Air Mobility (UAM) operations, reducing congestion and structural constraints are key challenges. Pioneering urban airspace design projects expect the air vehicles to fit into structured UAM corridor networks. However, most existing air transport networks are not capable of handling the increasing traffic demand, which is likely to cause congestion, traffic complexity, and safety issues. To adapt the increasing demand to the current airspace capacity, a novel macroscopic traffic assignment model is proposed to mitigate the congestion and organize the structure of air traffic flow. Firstly, the UAM corridor is designed and fitted into graph representation. Then, a traffic assignment problem based on Linear Dynamical System (LDS) is formalized to minimize the congestion factors and the intrinsic air traffic complexity. A two-step resolution method based on Dafermos' algorithm is introduced to efficiently solve this optimization problem. A case study is carried out on a two-layer air transport network with intensive UAM operations. The results demonstrate that the proposed model can successfully mitigate urban airspace congestion and organize the UAM traffic into a low-complexity flow pattern. This approach can be used as a tool to assist Air Navigation Service Provider (ANSP) in strategic planning for a given transportation network.

\footnotetext{
*PhD candidate, Optim group, ENAC, 7 Avenue Edouard Belin, zhengyi.wang@enac.fr

†Professor, Head of Optim group, ENAC, 7 Avenue Edouard Belin, delahaye@ recherche.enac.fr

${ }^{\ddagger}$ Research scientist, Head of Intelligent Systems and Decision reasearch unit, ONERA, 2 Avenue Edouard Belin, jean-loup.farges@onera.fr

${ }^{\S}$ Associate Professor, Deputy Director of ATMRI and Co-Director of SAAB-NTU Joint Lab, NTU, 50 Nanyang Avenue, sameeralam@ntu.edu.sg
} 


\section{Nomenclature}

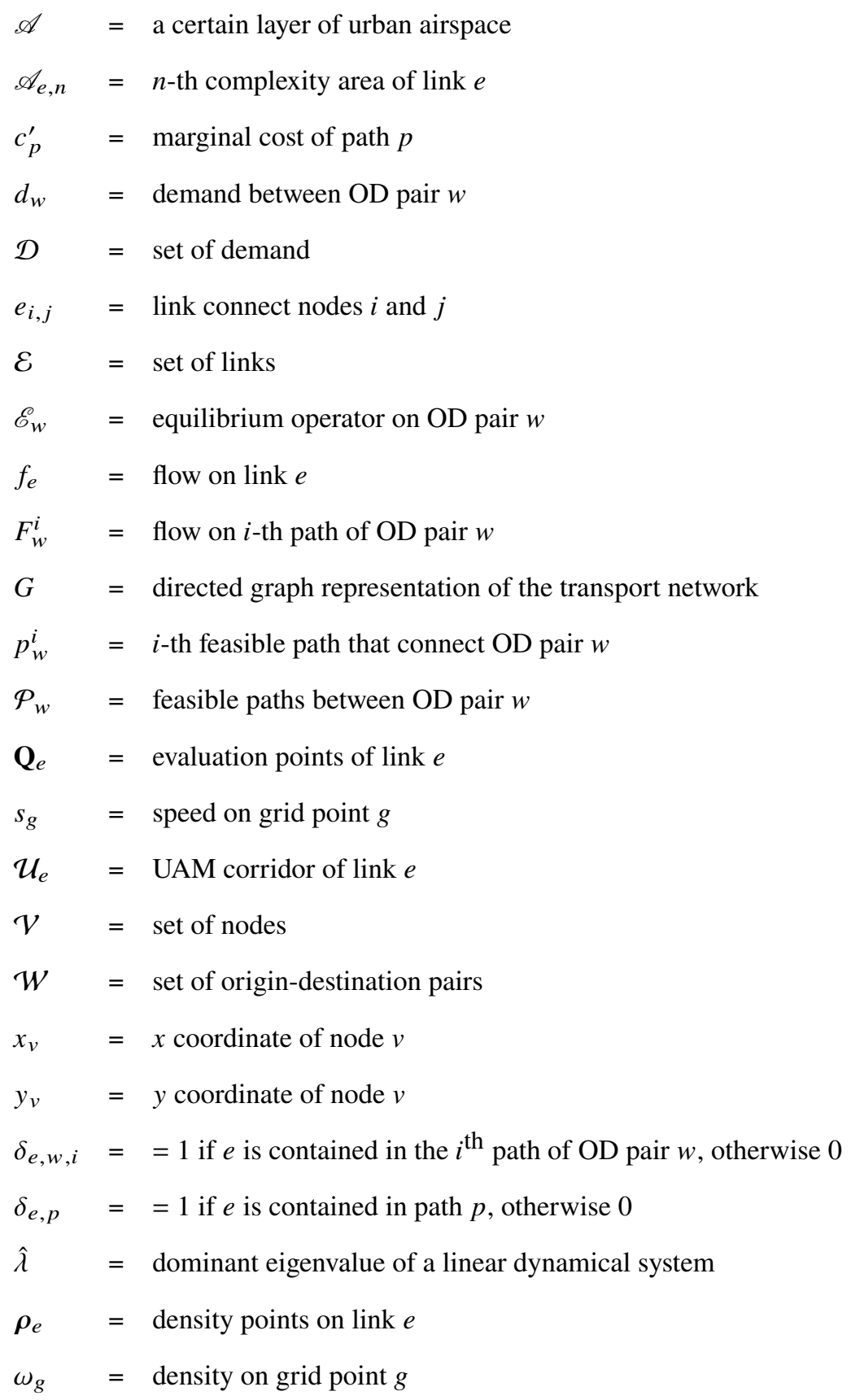

\section{Introduction}

Urban Air Mobility (UAM) is defined as air traffic operations to ensure safety and efficiency for manned and unmanned aircraft within a metropolitan area [1]. To integrate UAM with the current Air Traffic Control (ATC) system, the urban airspace system should be safe, efficient, and predictable, with minimum impact on the existing airspace operations [2]. Figure 1 presents some preliminary urban airspace design projects conducted by the TU Delft [3], the 
U.S. Federal Aviation Administration (FAA) [4], and the Amazon [5]. A common approach in these projects is to divide the urban airspace into several airspace classes and layers. Further, UAVs must operate in permitted airspace classes and fly below a certain altitude to keep away from planes, helicopters, and other manned vehicles. In addition, a novel route segment structure: UAM corridor was introduced to integrate UAM operations with Unmanned aircraft system traffic management (UTM) and Air Traffic Management (ATM) in urban airspace. Aircraft inside UAM corridors should follow UAM specific rules, procedures, and performance requirements. With the development of Vertical Takeoff and Landing (VTOL) or ultra-Short Takeoff and Landing (uSTOL), the main research challenge lies in mitigating the congestion and structuring the UAM operations in 2D planar transport networks in different flight levels [6].

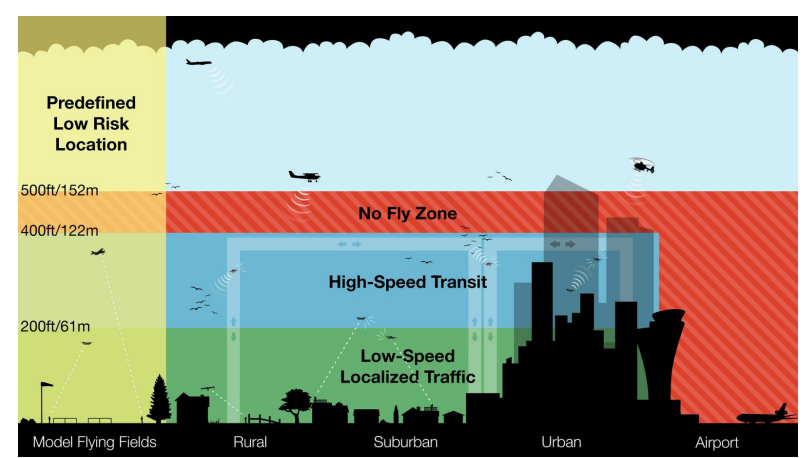

(a) Amazon [5]

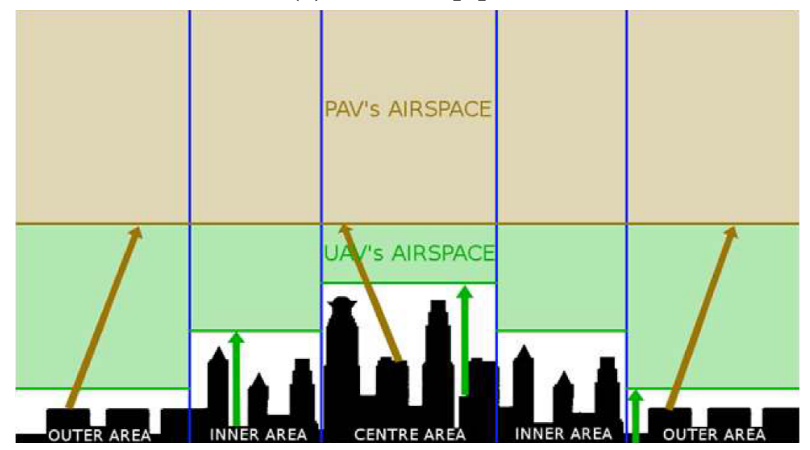

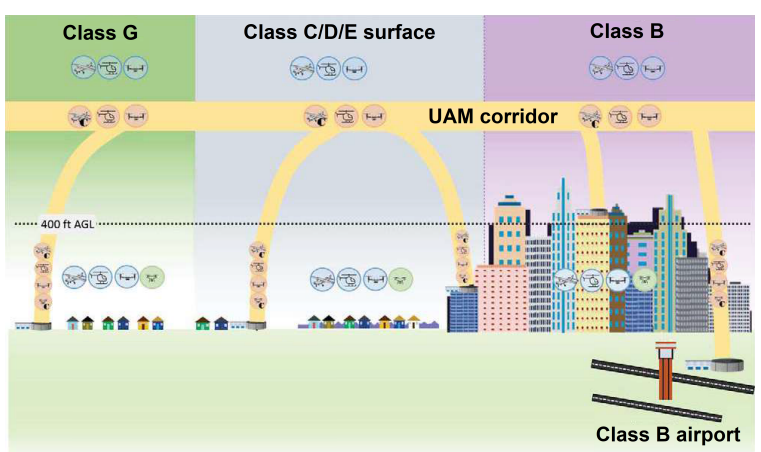

(b) ConOps, FAA [4]

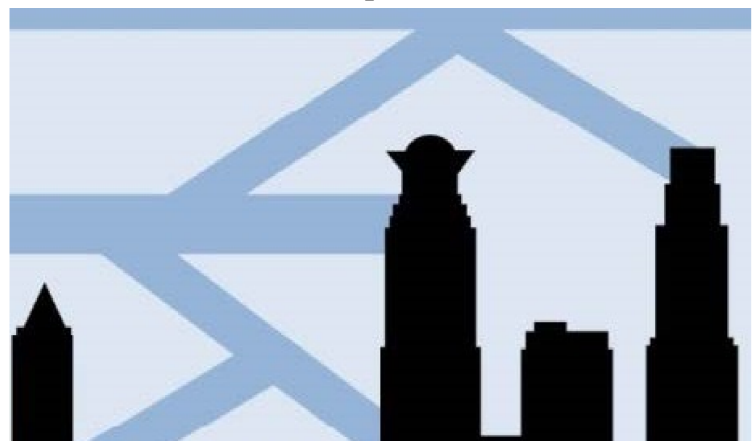

(c) Metropolis project, TU delft [3]

Fig. 1 Some preliminary urban airspace design projects

According to Metropolis project [7], aerial vehicles are expected to replace 1/6 of all traffic by 2050. In the near future, it is highly likely that the high-density traffic in urban airspace will exceed the capacity of the current urban airspace structure. In that case, existing air transport networks could bring congestion and increased traffic complexity, even safety and security issues such as potential conflicts [8]. Strategic air traffic planning is required to build smooth and efficient air transportation systems by reducing the airspace complexity and structuring air traffic. Though potential interactions or conflicts between aircraft may still exist, the separation of operations can be assured through various tactical conflict resolution methods within UAM corridors [9]. Moreover, these remaining conflicts and interactions will be much easier to solve when the airspace complexity is reduced and the traffic flow becomes more organized [10]. 
Theoretically, air transport enables greater freedom of route choices than most other transport modes. When a flight plan is assigned to aircraft, some flights may choose the same route (due to similar cost index based on shortest route) without considering the global optimum of the system. Such route choices may lead to conflicts as well as frequent congestion on commonly flown routes. To avoid such situations, air traffic assignment is widely used to search for a compromise between aircraft and airspace perspective in a way that not too many aircraft are penalized. Considerable research has been conducted into solving road traffic congestion to minimize a user or system criterion by distributing demand over different routes. A common approach in the literature is to propose the form of the link cost functions, then the parameters are derived from traffic and road conditions. Another type of cost function is developed on the basis of queuing theory [11]. Moreover, a vast majority of research on ground transportation system assumes that the link cost depends directly on the flows of this link and indirectly on other links in the surrounding area [12--16]. Some other factors are also found to be influential in the route assignment process, such as distance [17, 18], emission [19, 20], safety [18, 21] and road facilities [22, 23].

However, to the best of our knowledge, these influential factors neglect the geometric characteristics of the network of urban airspace and consider the structured and disordered traffic patterns equally. In this manner, a network may continue to accept structured traffic flow even though the operational capacity has been exceeded, and may refuse disordered traffic flow even if there is still enough operational capacity. Besides, the mixing level of aircraft trajectories also reflects inter-dependency between conflicts and the difficulty to manage the airspace [24]. As an intrinsic complexity measurement, through analysis of the airspace geometry and traffic structure, Linear Dynamical System (LDS) are also used in the literature [25]. A dynamical system describes the time dependence of an ensemble of particles in a geometrical space. The system behavior can be predicted for a short time into the future. Due to its relative simplicity, efficiency, and mathematically predictable behavior, LDS is quite suitable as a metric to estimate the local disorder and interaction of a set of trajectories in a traffic system.

This study focuses on strategic UAM operational planning through mitigating the congestion and organizing the flow structure for transport networks that consist of UAM corridors from a macroscopic perspective. Based on the aforementioned discussion, the contributions of this paper are 3-fold: (1) a flow dependent air traffic complexity criterion is developed from the air traffic complexity indicator based on trajectory measurement and linear dynamical system; (2) A novel complexity-optimal air traffic assignment model is developed from the macroscopic point of view; and (3) the problem is efficiently solved using a two-step resolution algorithm based on Dafermos' algorithm.

The remainder of this paper is organized as follows. The next section presents some related works with respect to complexity metrics and air traffic assignment. Section [II] introduces the mathematical model of air traffic assignment based on LDS in a planar UAM air transport network. In section[IV] an efficient two-step resolution algorithm for this optimization problem based on Dafermos' algorithm is proposed. Section $\square$ conducts case studies to demonstrate the proposed approach. Section $\mathrm{VI}$ summarizes the study and points out some future research directions. 


\section{Related works}

\section{A. Air traffic complexity metrics}

Air traffic complexity is referred to as a measure of the difficulty, for the system in-charge of traffic, to manage a situation in a particular airspace. It is widely used in Air Traffic Management (ATM) as it is a critical component of the airspace optimization process [26]. Precursory studies towards air traffic complexity emerged in the 1960s [27, 28] and continued to develop since then. As stated by Meckiff et al. [29], air traffic complexity consists of three elements: the geometric nature of air traffic, the operational procedures and practices used to handle the traffic, and the characteristics as well as the behavior of traffic control system involving human operators or automatic processes. These elements can be further refined as control workload and traffic complexity.

Control workload reflects the difficulty of a traffic control system to treat a traffic situation. Many previous related works pay particular attention to model the control workload on the provision of traffic situations, such as workload models based on the traffic level [30], airspace structure [31], queuing theory [32], traffic flow [33] and dynamic density [34, 35]. Several attempts have been made to model the workload of the traffic control system by automatic conflict resolution algorithms [26, 36, 37], in which the control workload is associated with the number of required modifications on trajectories for conflict resolution. To further evaluate the operational differences between the airspace structures, metrics based on environmental and operational factors are proposed [38]. All these models are highly dependent on the control systems or the algorithms involved. In addition, most of these metrics require a large amount of data during a long period, which has poor generalization property to new sectors.

Traffic complexity is an intrinsic measurement of the complexity involved in a traffic situation. It is independent of the traffic control system and only dependent on the geometry of trajectories. Typical metrics include geometric metrics [25], proximity metric, convergence metric [25], clusters metric [39], Grassmannian metric [40], König metric [41-43]. These basic geometrical metrics are suitable for capturing different kinds of features in terms of complexity. Gathering them together in some way can be much more powerful. Nevertheless, when a traffic situation has a more complex structure, these geometric metrics would fail. Besides, the evaluation of complexity only represents the current situation and cannot reflect the inter-dependency of future time. To overcome these issues, metrics based on dynamical systems are used to quantify the disorder, interaction, and evolution between trajectories in an airspace [25]. Linear Dynamical System has been proved to be suitable and effective for intrinsic air traffic complexity estimation in many studies [24, 25, 42, 44,-47].

\section{B. Air traffic assignment}

The airspace becomes more and more congested as the air traffic demand continues to increase. There are two main types of de-congestion strategies [48]. The first strategy adapts the capacity to the ever-increasing demand by re-configuring and extending the infrastructure of airspace, such as constructing new airports, extending the air transport 
network, increasing the number of controllers. However, airspace infrastructure is too costly to be extended on a wider scale, and moreover the airspace is limited [49]. The second de-congestion strategy is to adapt the demand to the current capacity, which aims to make use of the airspace more efficiently. One of the most widely-used methods is trajectory planning. Its principle is to generate a geometric path from origin to destination through predefined waypoints. In view of different phases, strategical or pre-tactical trajectory planning aims to resolve potential conflicts and alleviate traffic complexity, and tactical trajectory planning consists of deconfliction in real operations in a very short time period [50]. It is worth noting that the vast majority of trajectory planning studies concentrate on the individual vehicle. Another method that belongs to the second strategy is the traffic assignment. It involves the allocation of routes for flows in transportation systems.

The first air traffic assignment model has been developed as early in 1954 [51]. This model addressed the assignment for a given fleet to carry an anticipated traffic load over several routes at the minimum cost. Since then, a variety of traffic assignment models are developed for solving routes and slot allocation problem [33, 52, 55], Air Traffic Flow Management (ATFM) [56-59], en-route network management [60, 61], etc. The air traffic assignment in urban airspace has also been widely studied. Extensive research has focused on UAV task assignment [62-65]. Some other studies have been carried out for different purposes: to increase the airspace capacity, reduce the noise level [66-69], and reduce emission [69-71].

However, few previous research conducts air traffic assignment from a macroscopic perspective, i.e., the distribution of flows involving streams of air vehicles. In addition, little attention has been paid to reduce the intrinsic air traffic complexity. To overcome these issues, in this paper, we propose a complexity-optimal air traffic assignment model based on LDS.

\section{Mathematical model of air traffic assignment within high-density UAM operations}

\section{A. Graph representation of air transport network}

In future high-density UAM operational environment, the most natural way to describe the air traffic is probably the transportation network [72]. Air transport network will support passenger, cargo, and other operations. In this case, the nodes stand for waypoints, the links stand for the air route segment. The paths stand for air routes connecting two nodes. Demands are associated with every pair of Origin-destination (OD), which give rise to a traffic pattern.

Consider a geographical area $\mathcal{A} \subset \mathbb{R}^{2}$ corresponded to a certain flight level in urban airspace. A transport network in $\mathcal{A}$ is built with a direct graph $G=(\mathcal{V}, \mathcal{E})$ with $\mathcal{V}$ being the set of nodes and $\mathcal{E}$ being the set of links. The average speed of aircraft on link $e$ is set as $s_{e}$. The link connecting nodes $i$ and $j$ is indexed as $e_{i, j}$. The coordinates of nodes are $\left\{\left(x_{v}, y_{v}\right), v \in \mathcal{V}\right\}$.

Let $\mathcal{W}$ be the set of OD pairs, in which each element $w=(O, D)$ represents the origin node $O \in \mathcal{V}$ and the destination 
node $D \in \mathcal{V} . \mathcal{P}=\left\{\mathcal{P}_{w}, w \in \mathcal{W}\right\}$ denotes the set of feasible paths for all OD pairs, where $\mathcal{P}_{w}=\left\{p_{w}^{1}, \ldots, p_{w}^{R_{w}}\right\}$ represents all feasible paths that connects a specific OD pair $w$. All possible paths without dead end and cycles $\mathcal{P}_{w}=\left\{p_{w}^{1}, \ldots, p_{w}^{R_{w}}\right\}$ between OD pairs $w \in \mathcal{W}$ are obtained based on depth-first search [73]. Moreover, we introduce two logical functions to characterize the relationship between link and path. The first type of logical function indicates the number of path and the OD pair it belongs to:

$$
\delta_{e, w, i}= \begin{cases}1 & \text { if } e \text { is contained in the } i^{\text {th }} \text { path of OD pair } w \\ 0 & \text { else }\end{cases}
$$

Regardless of the detail of OD pair, the logical function of the second type only reflects the relationship between a link and a path:

$$
\delta_{e, p}= \begin{cases}1 & \text { if } e \text { is contained in path } p \\ 0 & \text { else }\end{cases}
$$

The demand between OD pairs is denoted by $\mathcal{D}=\left\{d_{w}, w \in \mathcal{W}\right\}$, which can be represented as a vector.

\section{B. UAM corridors design}

UAM corridor is an emerging concept defined by Administration [4], which refers to a route segment that connects two aerodrome locations to support point-to-point UAM operations without tactical ATC separation services [74]. UAM corridors are seen as a primary mechanism to integrate safe and efficient UAM operations in urban airspace. The impact on existing ATM and UTM operations can be reduced. Besides, public interest stakeholder needs (e.g., environmental factors, congestion, safety, security) and stakeholder utility (e.g., customer need) can also be addressed. Figure 2 illustrates some planar UAM corridors of different flight levels. For link $e \in \mathcal{E}$, the related UAM corridor $\mathcal{U}_{e} \in \mathcal{A}$ can be designed as a rectangle route segment with the same length as link $e$. To adapt UAM corridors in high-density operational perspective, two important terms density points and complexity area are introduced:

\section{Density points}

To describe the traffic density of UAM operations in a macroscopic perspective, density points are introduced to represent the flow distribution along the UAM corridor. A set of density points $\rho_{e}$ is defined as virtual grids located on each UAM corridor. Each density point $g$ has not only coordinates $\left(x_{g}, y_{g}\right)$ but also a heading $\theta_{e}$ and a speed $s_{e}$ inherited from the link $e$. Certain numbers of aircraft are considered at each point, which is also referred to as density and is dependent on the link flow. For every longitudinal position, the lateral density points are assumed to follow a uniform distribution of flow. Let the number of points on each side of the link be $N_{s}$. In order to equally share the flow 


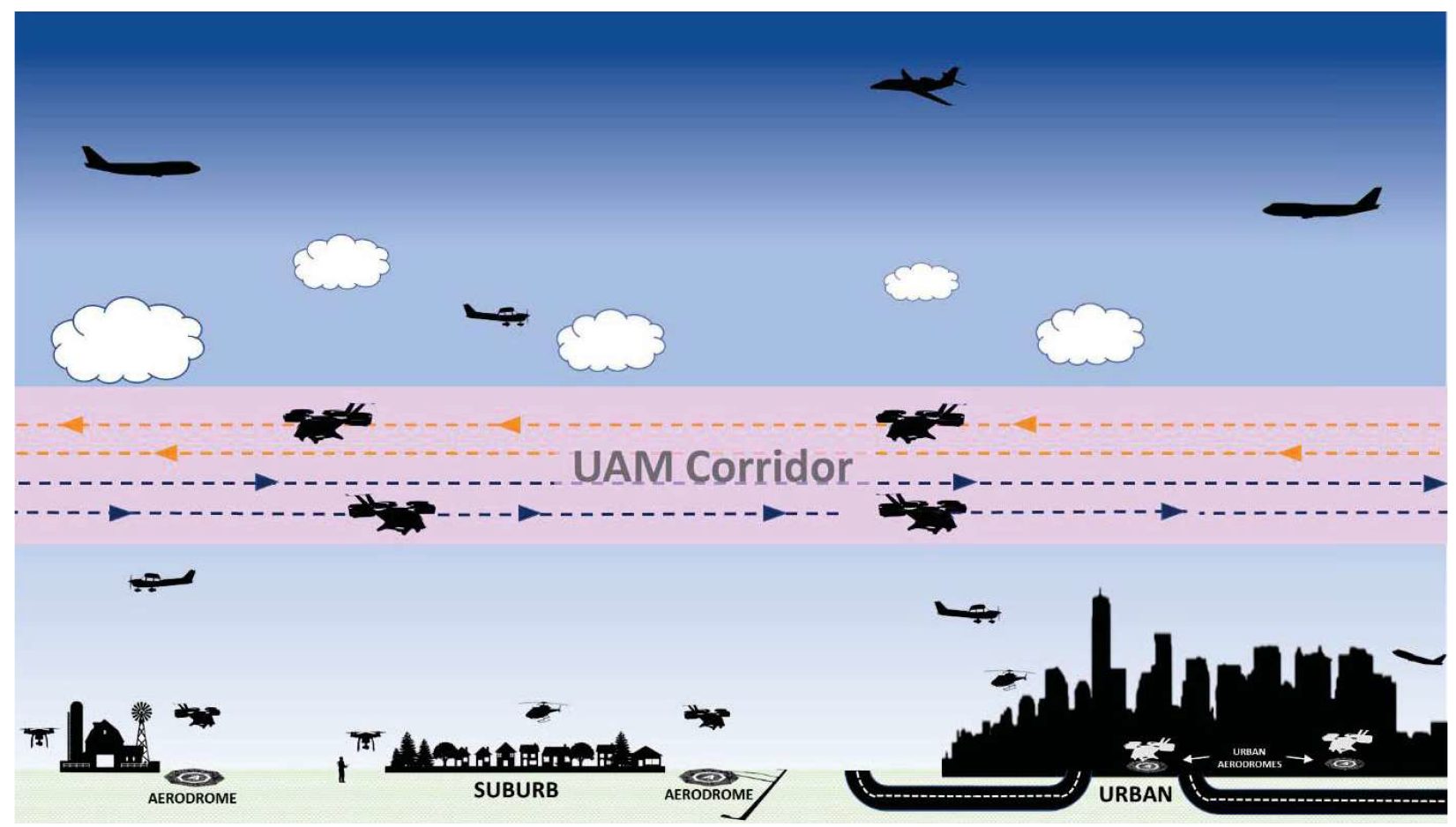

Fig. 2 UAM corridor integrated in different airspace classes [4]

in the lateral direction, the lateral flow sharing coefficient $\alpha_{e}$ is thus defined as:

$$
\alpha_{e}=\frac{1}{2 N_{s}+1}
$$

Due to the varying length of the UAM corridor, the distance of successive density points is not possible to be the same in the longitudinal direction. An alternative grid partition approach is formalized as Algorithm 1. For all UAM corridors, the size of grids is firstly assumed as the same and denoted as $L_{l} \times L_{w}$. The objective is to set the longitudinal distance between successive density points to $L_{l}$ with the exception that one longitudinal distance in the middle of the link belongs to $\left[L_{l}, 2 L_{l}[\right.$, which corresponds to lines 4 to 11 in Algorithm 1 . Though it is possible to distribute this excess space around the margin of the UAM corridor, the choice of concentrating the excess space in the middle of the link is more justified because it is the area where the complexity is likely to be the lowest and thus the bias is the smallest. To facilitate the computation, the grid partition is firstly conducted on a predefined coordinate system then translated and rotated to the original direction, which corresponds to Figure 3 and lines 3 and 14 in the pseudocode.

Finally, following the uniform distribution of flow, the speed $s_{g}$ for all density points in $\rho_{e}$ is set to the same value $s_{e}$. 

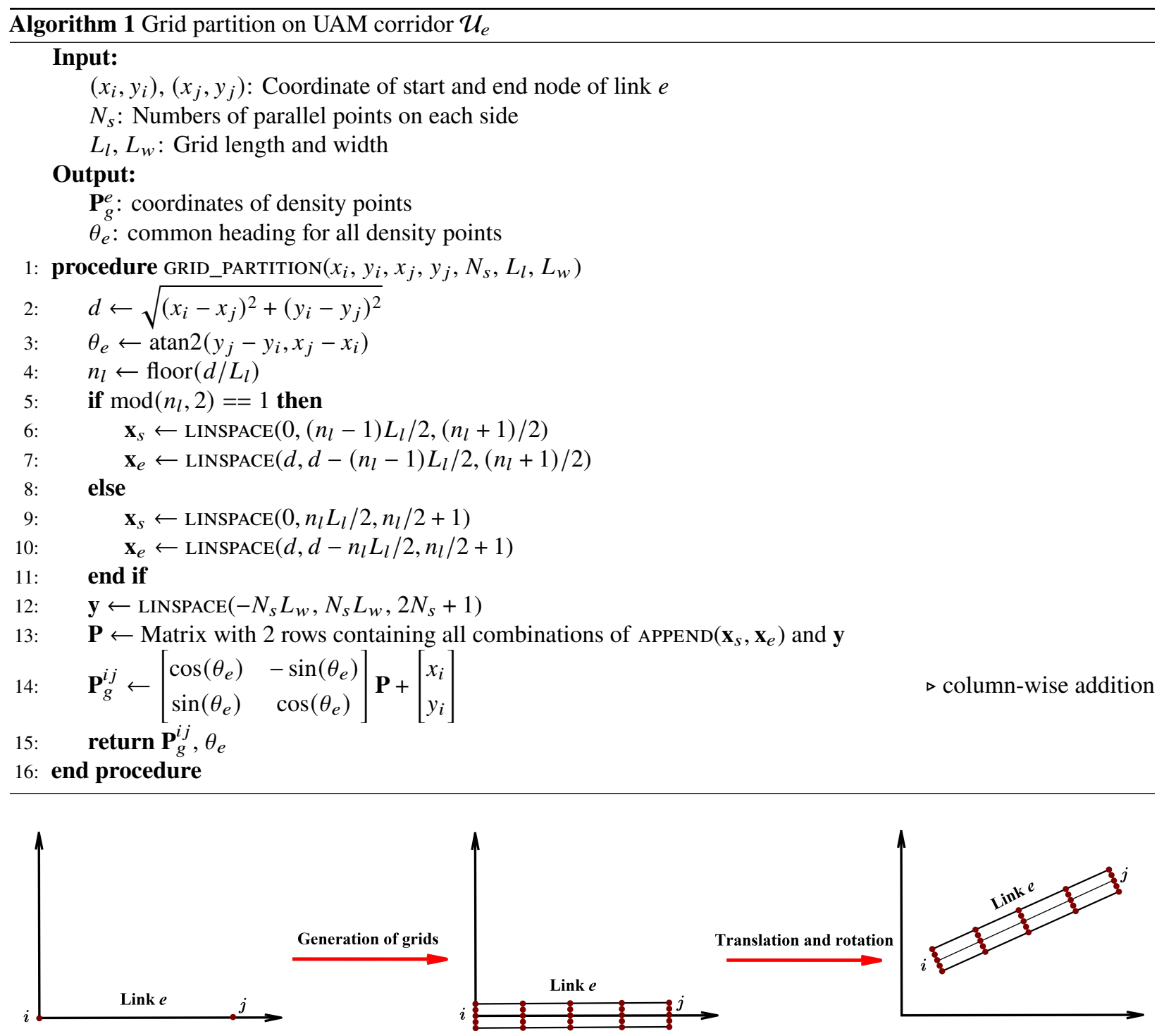

Fig. 3 Illustration of algorithm 1

\section{Complexity area}

In order to measure the air traffic complexity of the UAM corridor, not only its traffic should be included, but also the surrounding airspace. The complexity evaluation airspace for each UAM corridor is called complexity area. For $\mathcal{U}_{e}, e \in \mathcal{E}$, the complexity areas $\left\{\mathcal{A}_{e, 1}, \ldots, \mathcal{A}_{e, N_{c}}\right\}$ are defined as disks with radius $R_{e}$, where $N_{e}$ is the number of complexity areas for $\mathcal{U}_{e}$. Each area $\mathcal{A}_{e, i}$ includes a set of density points $\boldsymbol{\rho}_{e, i}$ with $\boldsymbol{\rho}_{e, i} \cap \boldsymbol{\rho}_{e} \neq \emptyset$. But in general, $\rho_{e, i} \not \subset \rho_{e}$ because the complexity area $\rho_{e, i}$ may include density points of other links $e^{\prime} \neq e$. Figure 4 illustrates a specific case, where the complexity areas are disks and the density points are located on grids aligned with the link. The centers of discal complexity areas are also longitudinally distributed between two nodes except for the extra space in the middle of the link. Those centers are referred to as evaluation points $\mathbf{Q}_{e}=\left\{q_{e, 1}, \ldots, q_{e, N_{c}}\right\}$ and can be determined by 
Algorithm 1 under $L_{w}=0$, and $N_{s}=0$. All points in the $i$-th complexity area of link $e, \forall q \in \mathcal{A}_{e, i}$, satisfies that the euclidean distance between $q$ and $q_{e, i}$ is not greater than the radius $R_{e}$. The set of links whose UAM corridors intersect with the complexity area $\mathcal{A}_{e, i}$ is denoted as $\mathcal{E}_{q_{e, i}}$. Figure 4 shows a small number of point-to-point UAM corridors. The complexity area of each UAM corridor is presented as a grey disk. From left to right, the complexity areas involve 4, 3, 4 links, respectively. The density points contained in each complexity area are specifically shown in details.

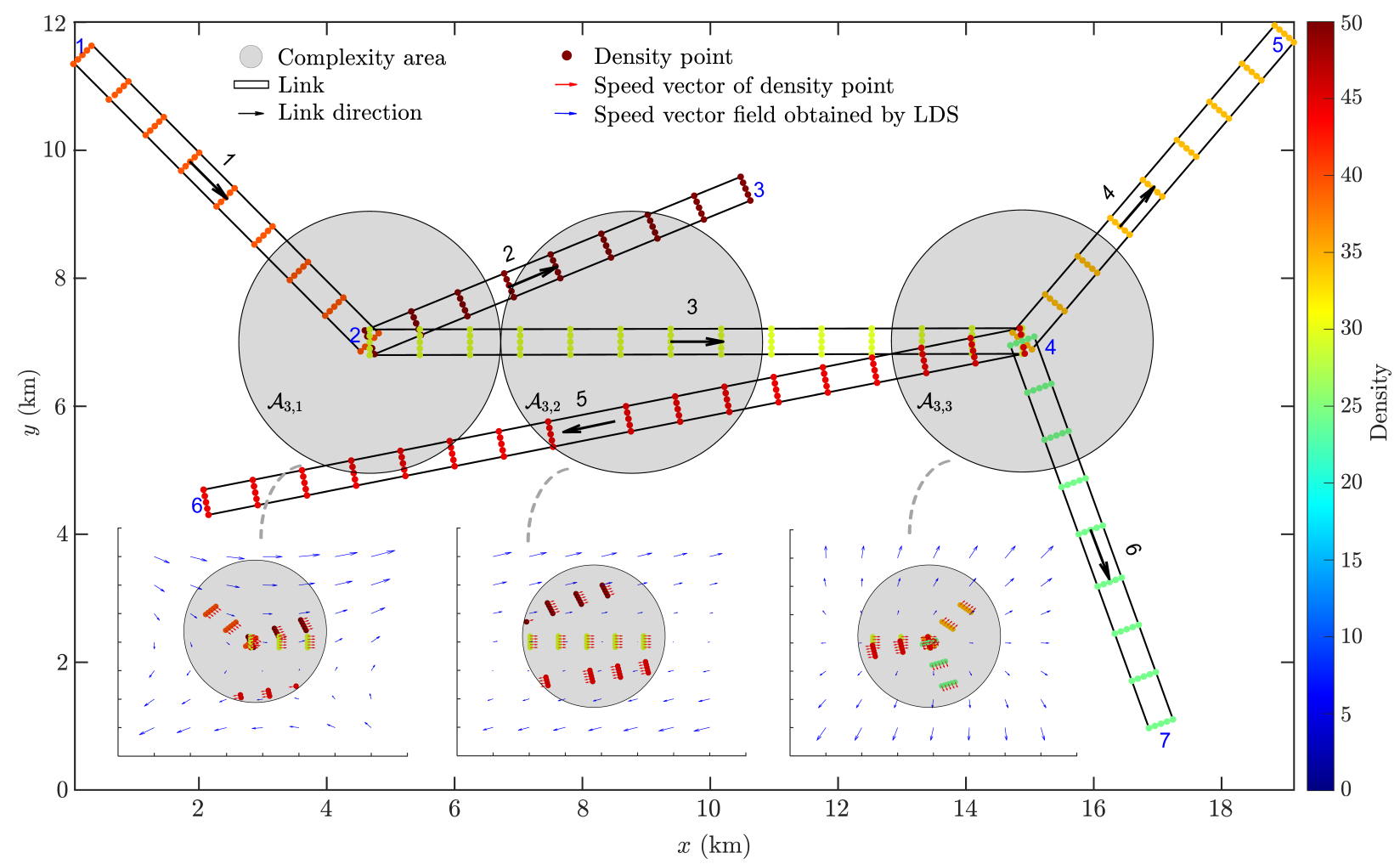

Fig. 4 Illustration of density points on UAM corridors, disk complexity complexity areas, and vector fields of LDS

\section{Optimization problem formulation}

The problem addressed in this paper can be stated as follows: given a transport network within UAM operations, the objective is to find the complexity-optimal flow distribution for UAM corridors such that the congestion and air traffic complexity of the transport network is minimized. In future UAM operations, air vehicles will follow the instructions delivered by external control systems, which involves cooperation between users. The total costs incurred to all users can be reduced to the minimum, corresponding to system equilibrium [75]. The optimization problem is then formalized under system equilibrium as follows: 


\section{Decision variables}

The flow on the feasible paths of $w$-th OD pair is denoted as $\mathbf{F}_{w}=\left\{F_{w}^{1}, \ldots, F_{w}^{R_{w}}\right\}$, which can be represented as a vector. The decision variables are flows $F_{w}^{i}$ on all paths indexed by $i \in\left\{1, \ldots, R_{w}\right\}$ of all OD $w \in \mathcal{W}$.

\section{Constraints}

For $w \in \mathcal{W}$, the path flows satisfies the conservation law:

$$
d_{w}=\sum_{i=1}^{R_{w}} F_{w}^{i}
$$

Moreover, the flow on all paths must not be negative:

$$
F_{w}^{i} \geq 0 \quad \forall w \in \mathcal{W}, \quad \forall i \in\left\{1, \ldots, R_{w}\right\}
$$

Given the flow of all admissible paths, the flow on a link $e \in \mathcal{E}$ is given by:

$$
f_{e}=\sum_{w \in \mathcal{W}} \sum_{i=1}^{R_{w}} \delta_{e, w, i} F_{w}^{i}
$$

To share the flow for density points, the traffic flow of aircraft within UAM corridor $\mathcal{U}_{e}$ is assumed with average speed $s_{e}$ and heading $\theta_{e}$. The density of each density point $g \in \mathcal{U}_{e}$ is defined as:

$$
\omega_{g}=\beta \frac{\alpha_{e} f_{e}}{s_{e}}, \quad \forall g \in \mathcal{U}_{e}
$$

where $\beta$ is a parameter to convert $\omega_{g}$ to a convenient unit and adjust the scale. The density is considered to be proportional to the flow $f_{e}$ and the lateral flow sharing coefficient $\alpha_{e}$, and inversely proportional to the average speed $s_{e}$.

\section{Objective function based on Linear Dynamical System}

To evaluate the air traffic complexity, the LDS is taken into account in the link cost function, in which more intrinsic information will be involved such as flow, information of density points, and configuration of nearby links around link $e$. In addition, LDS is able to quantify the disorder, interaction, and evolution between trajectories in the airspace. As a strategical planning model, when the complexity metric based on LDS becomes lower, the flow will become more organized, the conflicts or interactions in real UAM operations will be reduced. The safety and efficiency of an autonomous UAM system can be guaranteed. In order to compute the complexity on the whole graph, the complexity of each complexity area $\mathcal{A}_{e, i}$ with $i \in\left\{1, \ldots, N_{c}\right\}$ and $e \in \mathcal{E}$ are computed using LDS. The density, position, heading, and speed of density points in each complexity area are used to determine the coefficient matrices and parameters of 
the corresponding LDS that best fits the density points. Then, the calculated dominant eigenvalue and the flow on the associated links can be used to construct the link cost function.

A LDS in geographical area $\mathcal{A}$ can be expressed as a differential equation of motion:

$$
\mathbf{v}=\mathbf{A x}+\mathbf{b}
$$

where $\mathbf{x}$ denotes the state vector of the system, $\mathbf{v}$ represents the speed vector, $\mathbf{A}$ stands for a coefficient matrix, $\mathbf{b}$ is a coefficient vector.

Conventional implementations of LDS simply concatenating the positions and speeds of all points in the complexity area $\mathcal{A}_{e, i}$ [25]. To calculate the error criterion much more efficiently, a weighted Minimum Mean Square Error (MMSE) estimator is proposed to adjust the parameters of LDS:

$$
E_{e, i}=\sum_{g \in \mathcal{A}_{e, i}} \omega_{g}\left\|\mathbf{v}_{g}-\left(\mathbf{A}_{e, i} \mathbf{x}_{g}+\mathbf{b}_{e, i}\right)\right\|^{2}
$$

where the density $\omega_{g}$, position vector $\mathbf{x}_{g}=\left(x_{g}, y_{g}\right)^{\top}$, heading $\theta_{g}$, speed $s_{g}$, and speed vector $\mathbf{v}_{g}=s_{g}\left(\cos \left(\theta_{g}\right), \sin \left(\theta_{g}\right)\right)^{\top}$ are inherited from the UAM corridor they belong to. It is worth noting that density points contained in a certain complexity area may be derived from one UAM corridor or multiple UAM corridors.

The complete calculation procedure of coefficient matrices of LDS is provided in VI.A After determining $\mathbf{A}_{e, i}$ and $\mathbf{b}_{e, i}$, LDS can generate a vector field that fits the observations with the minimum weighted MMSE. For instance, three detailed coordinate systems in the bottom of Figure 4 illustrates the vector fields generated by LDS based on density points in each complexity area. The arrow length is proportional to the velocity. The color of density points corresponds to values in the colorbar. It can be seen that the goodness of fit in terms of the vector field generated by LDS is quite satisfying.

The matrix $\hat{\mathbf{A}}_{e, i}$ that minimizes the MMSE can be derived. Let $\lambda_{e, i}^{1}$ and $\lambda_{e, i}^{2}$ be the two eigenvalues of $\hat{\mathbf{A}}_{e, i}$ respectively. The convergence or the divergence property of the system can be determined in terms of the eigenvalues. Eigenvalue with a positive real part corresponds to a system in expansion mode. If the real part is negative, the system is in contraction mode. The absolute value of the real part represents the level of contraction or expansion of the system. Besides, the imaginary part of the eigenvalues, $\operatorname{Im}\left(\lambda_{e, i}^{1}\right)$ and $\operatorname{Im}\left(\lambda_{e, i}^{2}\right)$, represents the curl organization of the system, i.e., the rotation tendency of the vector field. In sum, an LDS can evolve in contraction, expansion, rotation, or the combinations. The basic evolution of LDS is presented in Figure 5 The vertical strip around the imaginary axis corresponds to organized traffic situations, where the eigenvalues of $\mathbf{A}_{e, i}$ have a real part close to zero. In these situations, the relative distances between points change slowly with time, namely, their relative speeds are close to zero and there is nearly no interaction between them. Furthermore, the eigenvalue loci for some typical traffic situations are 
discussed in section VI.B

In the proposed model, the convergence and divergence cases are both considered as unorganized structures. The dominant eigenvalue $\hat{\lambda}_{e, i}$ is then proposed to evaluate the evolution tendency of the system. It is defined as the largest absolute value of real part among the two eigenvalues:

$$
\hat{\lambda}_{e, i}=\max \left(\left|\operatorname{Re}\left(\lambda_{e, i}^{1}\right)\right|,\left|\operatorname{Re}\left(\lambda_{e, i}^{2}\right)\right|\right)
$$

The absolute value operation unifies the convergence and divergence cases. The more the value of the dominant eigenvalue is closer to zero, the more traffic situation is organized.

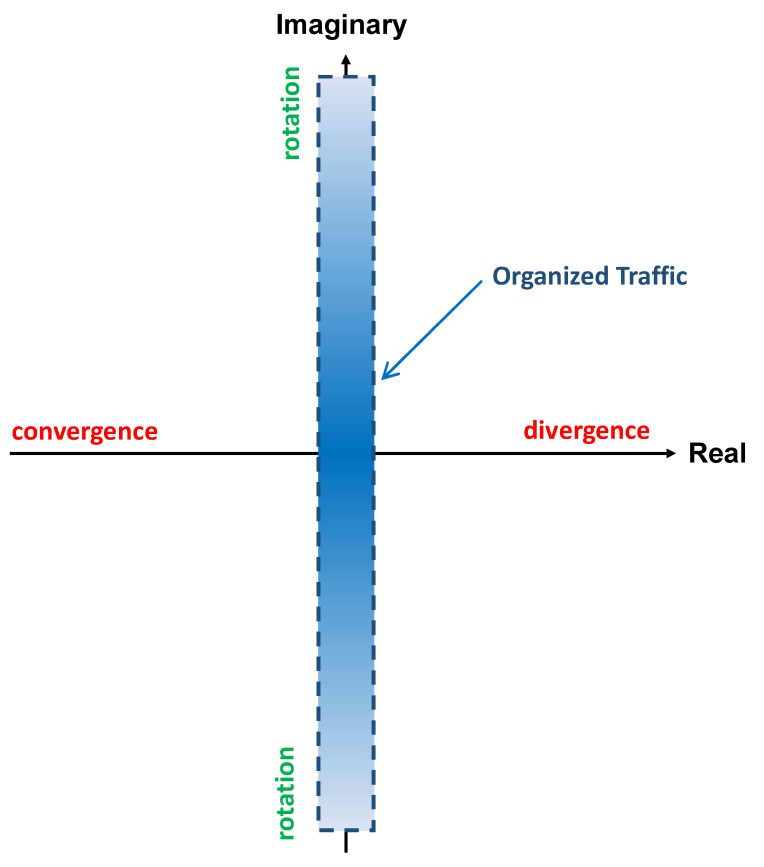

Fig. 5 Evolution in terms of eigenvalues

To involve the air traffic complexity measurement defined by LDS and the traffic flow of neighboring links, the cost for link $e$ is designed as:

$$
c_{e}=\frac{1}{N_{e}} \sum_{i=1}^{N_{e}} \hat{\lambda}_{e, i} \sum_{e^{\prime} \in \mathcal{E}_{q_{e, i}}} f_{e^{\prime}}
$$

This cost function corresponds to the weighted sum of the dominant eigenvalue of LDS in each complexity area multiplied by the sum of flow for evaluated links. That is, the complexity is weighted by the flow and involved in the complexity area. 
Finally, the optimization problem can be formalized as follows:

$$
\begin{aligned}
\min _{\mathbf{f}=\left\{f_{e} \mid e \in \mathcal{E}\right\}} & S(\mathbf{f})=\sum_{e \in \mathcal{E}} \frac{1}{N_{e}} \sum_{i=1}^{N_{e}} \hat{\lambda}_{e, i} \sum_{e^{\prime} \in \mathcal{E}_{q_{e, i}}} f_{e^{\prime}} \\
\text { s.t. } & f_{e}-\sum_{w \in \mathcal{W}} \sum_{i=1}^{R_{w}} \delta_{e, w, i} F_{w}^{i}=0, \quad e \in \mathcal{E} \\
& F_{w}^{i} \geq 0, \quad w \in \mathcal{W}, i \in\left\{1, \ldots, R_{w}\right\} \\
& \sum_{i=1}^{R_{w}} F_{w}^{i}=d_{w}, \quad w \in \mathcal{W}
\end{aligned}
$$

\section{Resolution algorithm}

Dafermos' algorithm is a efficient sequential decomposition algorithms for traffic assignment [76-78]. It is designed to determine the flow patterns under system equilibrium in a transport network. Given path $p$ as the $i^{\text {th }}$ path of $w$, the marginal cost $c_{p}^{\prime}$ is defined as follows:

$$
c_{p}^{\prime}=\frac{\partial S}{\partial F_{w}^{i}}=\sum_{a, b \in \mathcal{E}} \frac{\partial c_{a}}{\partial f_{b}} \delta_{b, w, i}
$$

Since the marginal cost for all paths with non-zero flow are equal when the system equilibrium is reached, this algorithm searches the most expensive route $p_{w, \max }$ and the least expensive route $p_{w, \min }$ in terms of marginal cost for each Origin-Destination (OD) pair. Then, a quantity of flow is transferred from $p_{w, \max }$ to $p_{w, \min }$ such that the global cost is minimized. This procedure is repeated for all OD pairs iteratively until the global cost cannot be further reduced. This algorithm determines the system-optimizing flow pattern by defining a family of operators $\mathscr{E}_{w}$ associated with the OD pairs $w \in W$ :

$$
\begin{aligned}
\mathscr{E}_{w}: \mathbb{Z} & \rightarrow \mathbb{Z} \\
\mathbf{F}_{w} & \mapsto \mathscr{E}_{w} \mathbf{F}_{w}
\end{aligned}
$$

The algorithm starts from an initial flow pattern $\left\{\mathbf{F}_{w}^{(0)}, w \in \mathcal{W}\right\}$. The global cost function converges by iterating the following operation:

$$
\mathbf{F}_{w}^{(n)}=\mathscr{E}_{w} \mathbf{F}_{w}^{(n-1)}, \quad n \in \mathbb{N}^{*}
$$


To construct $\mathscr{E}_{w}$, the least and most expensive routes are firstly determined:

$$
\begin{aligned}
& p_{w, \text { min }}=\underset{p \in \mathcal{P}_{w}}{\arg \min } c_{p}^{\prime} \\
& p_{w, \max }=\underset{p \in \mathcal{P}_{w}, F_{p}>0}{\arg \max } c_{p}^{\prime}
\end{aligned}
$$

The cost function depends not only on the flow pattern, but also on the air traffic complexity metric, which is the dominant eigenvalue of the LDS in terms of complexity areas. The representation of the cost function makes its derivative very complicated. Numerical differentiation techniques has been adopted to estimate the marginal cost. For $a, b \in \mathcal{E}$, the derivative of $c_{a}$ with respect to $f_{b}$ is calculated by finite difference approximations:

$$
\frac{\partial c_{a}}{\partial f_{b}}=\lim _{h \rightarrow 0} \frac{c_{a}\left(f_{b}+h\right)-c_{a}\left(f_{b}\right)}{h}
$$

where $h$ represents a small variation in $f_{b}$. It is noteworthy that according to Equation (7), $f_{b}$ is proportional to $\omega_{g}$. Since the density $\omega_{g} \in \mathbb{R}^{+}$make sense in the weighted form of Equation 9, $h$ can be taken as sufficiently small as possible.

Then, the new flow on paths $\hat{\mathbf{F}}_{w}=\left\{\hat{F}_{p}^{1}, \ldots, \hat{F}_{p}^{R_{w}}\right\}$ are updated as the following distribution rule:

$$
\begin{aligned}
& \hat{F}_{p}=F_{p}, \quad \forall p \in\left(\mathcal{P}_{w}-\left\{p_{w, \min }\right\} \cup\left\{p_{w, \max }\right\}\right) \\
& \hat{F}_{p_{w, \text { min }}}=F_{p_{w, \min }}+\sigma_{w} \\
& \hat{F}_{p_{w, \max }}=F_{p_{w, \max }}-\sigma_{w}
\end{aligned}
$$

where $\sigma_{w}$ is the solution to the given optimization problem:

$$
\begin{array}{ll}
\min _{\sigma_{w}} & \mathscr{S} \\
\text { s.t. } & f_{e}^{\prime}=f_{e}+\left(\delta_{a, p_{w, \text { min }}}-\delta_{a, p_{w, \max }}\right) \sigma_{w}, \quad e \in \mathcal{E} \\
& \sigma_{w} \in\left[-F_{p_{w, \text { min }}}, F_{p_{w, \text { max }}}\right]
\end{array}
$$

The whole process of an iteration of Dafermos' algorithm is illustrated on a simple network in Figure $6 \mathrm{a}$

Based on [76, 78], the analytical representation of $\sigma_{w}$ was easily computed with the assumption of a quadratic form of the cost function. For the sake of convergence, the overall global cost should be convex with regards to link flows. In our problem, the dominant eigenvalue is involved in the cost function and it is dependent with link flow. It could be very difficult to derive the exact mathematical derivation of the cost function, which cannot assure the convexity of cost function. Besides, the scale of dominant eigenvalues depends on the traffic situations, which may result in large 


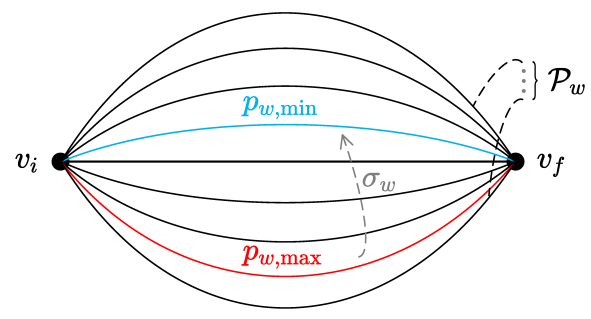

(a) Conventional Dafermos' algorithm

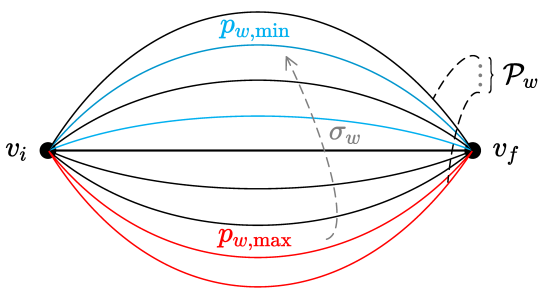

(b) Modified Dafermos' algorithm

Fig. 6 The process of an iteration of conventional and the modified Dafermos' algorithm

differences between the marginal costs of each path. The path with the maximum or minimum marginal cost may not change after transferring the flow, making the whole algorithm get stuck in the local optimum. To this end, we propose a two-step strategy to improve the Dafermos' algorithm. The flowchart of this approach is presented in Figure 7 The first step follows equations (18) - (24) and corresponds to operator $\mathscr{E}_{w, 1}$. The brute-force approach is used to solve the optimization problem [23). All values of $\sigma_{w}$ in the interval are evaluated and the value that makes $\mathscr{S}$ minimum is chosen. The convergence speed is very fast until the local optimum is reached. If the global cost function stops decreasing, the second step associated with operator $\mathscr{E}_{w, 2}$ starts by following equations (21) - (28). The second step determines the paths $p_{w, \min }, p_{w, \max } \in \mathcal{P}_{w}$ in an alternative way:

$$
\begin{aligned}
& p_{w, \text { min }}=\operatorname{random}\left(\underset{p \in \mathcal{P}_{w}}{\arg \min }\left(c_{p}^{\prime}, N_{p_{w, \text { min }}}\right)\right) \\
& p_{w, \text { max }}=\operatorname{random}\left(\underset{p \in \mathcal{P}_{w}, F_{p}>0}{\arg \max }\left(c_{p}^{\prime}, N_{p_{w, \text { max }}}\right)\right)
\end{aligned}
$$

where Equation 24] and 25] are defined to return a random path from $N_{p_{w, \text { min }}}$ smallest argument and $N_{p_{w, \text { max }}}$ largest argument of marginal cost under given conditions, respectively. A possible way to determine the values of $N_{p_{w, \min }}$ and $N_{p_{w, \max }}$ can be:

$$
\begin{aligned}
& N_{p_{w, \text { min }}}=\min \left(R_{w}-1, N_{r}\right) \\
& N_{p_{w, \text { max }}}=\min \left(\operatorname{card}\left(\left\{p \mid F_{p}>0, p \in \mathcal{P}_{w}\right\}\right)-1, N_{r}\right)
\end{aligned}
$$

where $R_{w}-1 \in \mathbb{Z}^{+}$and $\operatorname{card}\left(\left\{p \mid F_{p}>0, p \in \mathcal{P}_{w}\right\}\right)-1 \in \mathbb{Z}^{+}$. These two variables control the lower limit of $N_{p_{w, \text { min }}}$ and $N_{p_{w, \max }} . N_{r}$ is a parameter to limit the maximum number of arguments to choose.

By this means, the paths $p_{w, \min }$ and $p_{w, \max }$ are randomly chosen from sets of paths with maximum and minimum marginal cost, rather than choosing paths with exact maximum and minimum marginal cost. It forces to transfer flow between paths, which helps escape from local optima. An iteration of the modified Dafermos' algorithm is depicted in Figure 6b given the random select number $N_{p_{w, \min }}=N_{p_{w, \max }}=2$. 


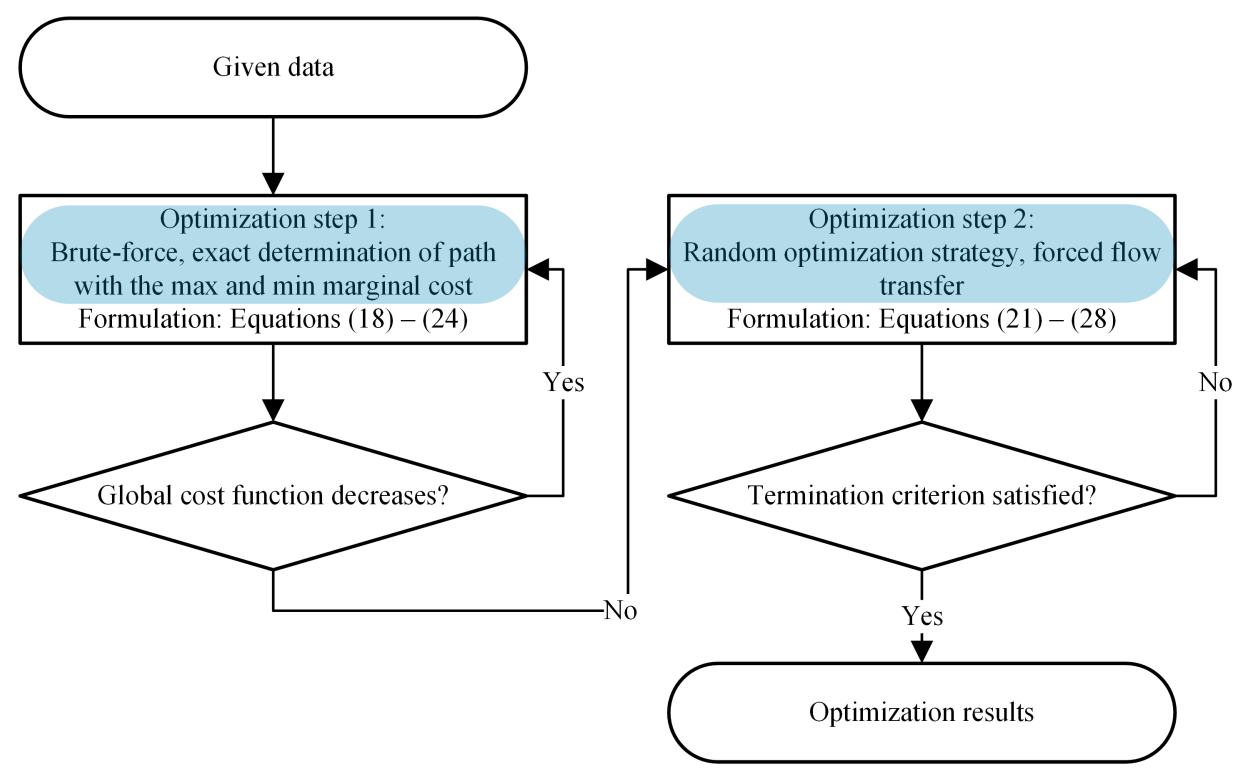

Fig. 7 The two-step optimization algorithm for our problem

To solve optimization problem (23), a Random Optimization (RO) strategy is used. As a numerical optimization method, this strategy is suitable for discontinuous and non-differentiable functions. The pseudocode is shown in Algorithm 2 and is also illustrated in Figure 8 . This approach proceeds $N_{t}$ epochs in each iteration, by moving to other positions in the search space and sampling with a given distribution $\mathscr{D}$ if it is a better position.

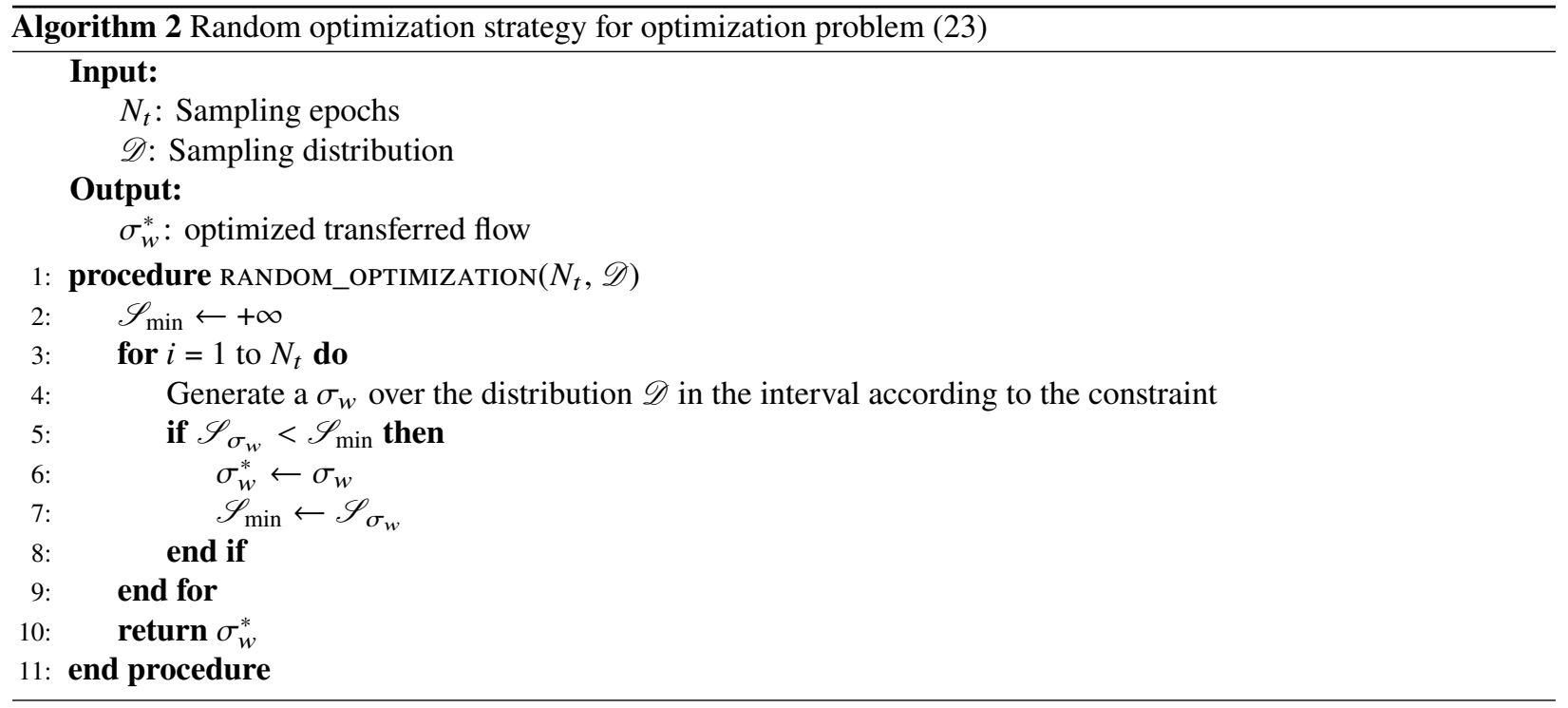

The whole resolution algorithm is terminated if any of the following three criteria are satisfied:

1) Limit on the number of iterations: when the total number of iterations exceeds the maximum number of iterations $N_{m}$.

2) Number of iterations without progress: when the number of iteration with no progress on the global cost function 


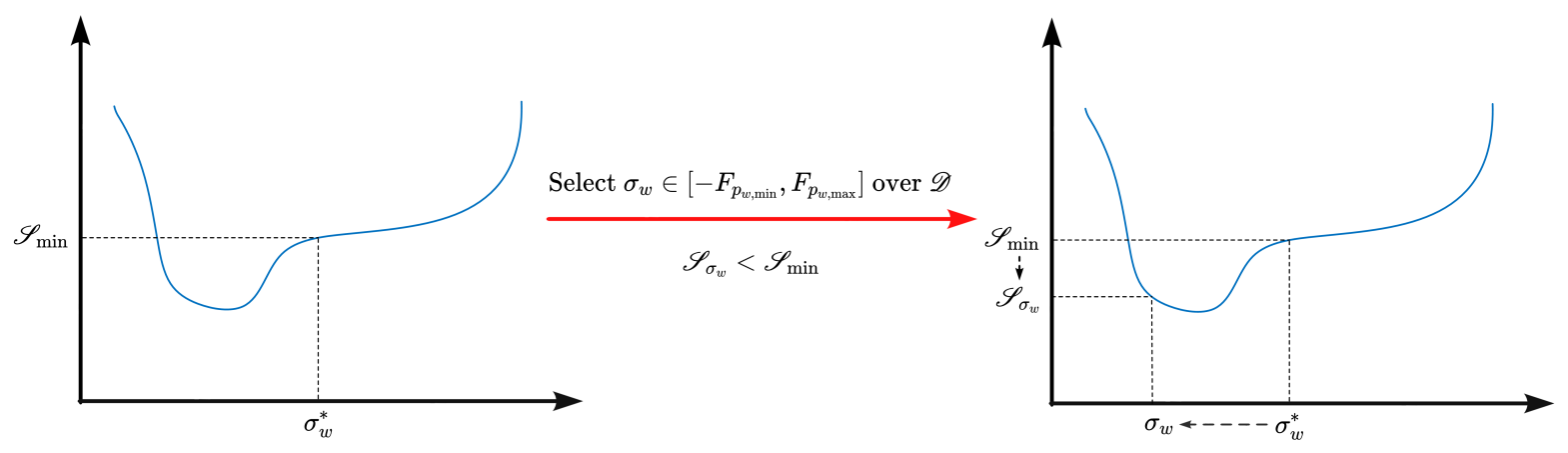

Fig. 8 Illustration of random optimization strategy

exceeds the number of patience $N_{p}$.

3) Convergence test: when the value of the global cost function is sufficiently small in some sense, lower than a threshold of $v_{s}$.

\section{Experiments}

\section{A. Case study}

To demonstrate the applicability and effectiveness of the proposed algorithm, two planar air transport networks are introduced for the experiment. The links in the same network never intersect except at the nodes. These two networks are denoted as $G_{1}$ and $G_{2} . G_{1}$ is a network modified on the basis of a transport network for last-mile delivery in Maribyrnong, Melbourne [79]. This transport network is able to cover the suburban and urban areas of most cities in the world. $G_{2}$ a real air transport network in ATM operations extracted from [60].

The coordinates of nodes, and the index of nodes and links are illustrated in Figure 9 The topological characteristics of $G_{1}$ and $G_{2}$ are summarized as follows:

- Set of nodes $\mathcal{V}_{1}=\left\{v_{1,1}, \ldots, v_{1,17}\right\} ; \mathcal{V}_{2}=\left\{v_{2,1}, \ldots, v_{2,24}\right\}$,

- Set of links $\mathcal{E}_{1}=\left\{e_{1,1}, \ldots, e_{1,28}\right\} ; \mathcal{E}_{2}=\left\{e_{2,1}, \ldots, e_{2,53}\right\}$

- Set of OD pairs $\mathcal{W}_{1}=\left\{w_{1,1}, w_{1,2}\right\}$ with $w_{1,1}=\left(v_{1,1}, v_{1,17}\right), w_{1,2}=\left(v_{1,4}, v_{1,7}\right) ; \mathcal{W}_{2}=\left\{w_{2,1}, w_{2,2}\right\}$ with $w_{2,1}=\left(v_{2,16}, v_{2,24}\right), w_{2,2}=\left(v_{2,1}, v_{2,22}\right)$.

The feasible paths are calculated for $G_{1}$ and $G_{2}$ :

- Set of feasible paths $\mathcal{P}_{1}=\left\{\mathcal{P}_{w_{1,1}}, \mathcal{P}_{w_{1,2}}\right\}$ with $\mathcal{P}_{w_{1,1}}=\left\{p_{w_{1,1}}^{1}, \ldots, p_{w_{1,1}}^{55}\right\}, \mathcal{P}_{w_{1,2}}=\left\{p_{w_{1,2}}^{1}, \ldots, p_{w_{1,2}}^{11}\right\} ; \mathcal{P}_{2}=$ $\left\{\mathcal{P}_{w_{2,1}}, \mathcal{P}_{w_{2,2}}\right\}$ with $\mathcal{P}_{w_{2,1}}=\left\{p_{w_{2,1}}^{1}, \ldots, p_{w_{2,1}}^{7}\right\}, \mathcal{P}_{w_{2,2}}=\left\{p_{w_{2,2}}^{1}, \ldots, p_{w_{2,2}}^{54}\right\}$

Within a high-density UAM operational scenario, the demands of each OD pair (aircraft/h) for $G_{1}$ and $G_{2}$ are set as:

- Set of demands $\mathcal{D}_{1}=\left\{d_{w_{1,1}}, d_{w_{1,2}}\right\}$ with $d_{w_{1,1}}=1925$ and $d_{w_{1,2}}=2255 ; \mathcal{D}_{2}=\left\{d_{w_{2,1}}, d_{w_{2,2}}\right\}$ with $d_{w_{2,1}}=1995$ and $d_{w_{2,2}}=2160$

The distribution of travel demands is initialized by means of distributing the demands equally among the allowable 

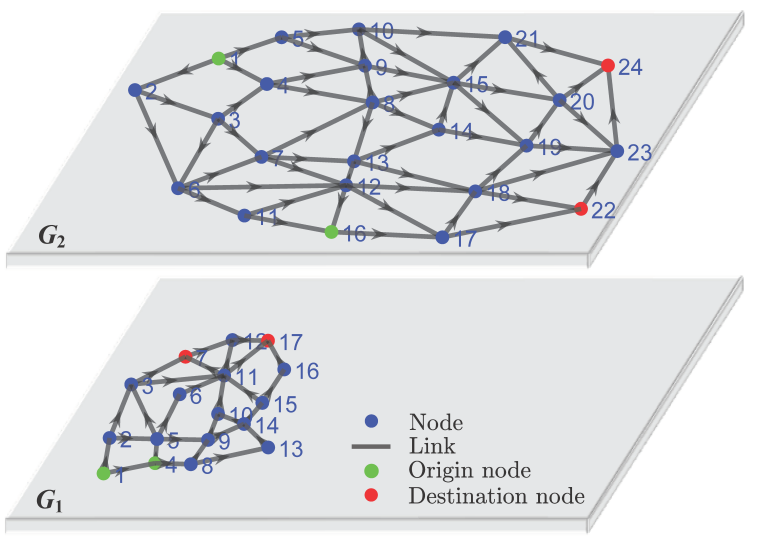

Fig. 9 Representation of the two-layer network, in which the first layer contains 17 nodes, 28 links and 2 OD pairs, and the second layer contains 24 nodes, 53 links and 2 OD pairs

Table 1 Parameters setting

\begin{tabular}{llll}
\hline Process & Parameter & Value & Description \\
\hline & $L_{l}$ & $2(\mathrm{~km})$ & Length of grid \\
& $L_{w}$ & $0.02(\mathrm{~km})$ & Width of grid \\
UAM corridors design & $s_{e}$ & $200(\mathrm{~km} / \mathrm{h})$ & Speed for link $e$ \\
& $N_{s}$ & 2 & Number of lateral points on each side \\
& $\beta$ & $10\left(\mathrm{~km}^{-1}\right)$ & Unit conversion coefficient \\
& $R_{e}$ & $5(\mathrm{~km})$ & Radius of complexity area for link $e$ \\
\hline & $h$ & 0.01 & Small variation for numerical derivation \\
& $N_{t}$ & 20 & Sampling epoch in RO strategy \\
& $N_{r}$ & 2 & Number of alternative suboptimal paths \\
& $\mathscr{D}$ & uniform distribution & Sampling distribution in RO strategy \\
& $N_{m}$ & 1000 & Total number of iteration \\
& $N_{p}$ & 200 & Number of iteration without progress \\
& $v_{s}$ & 100 & Target value of criteria \\
\hline
\end{tabular}

Note: $e \in \mathcal{E}_{1} \cup \mathcal{E}_{2}$

paths. For $w \in \mathcal{W}_{1} \cup \mathcal{W}_{2}$,

$$
F_{w}^{i}=\frac{d_{w}}{R_{w}}, \quad \forall i=1, \ldots, R_{w}
$$

The parameters used in UAM corridors design and resolution algorithm for optimization problem (23) are respectively given in two parts of Table 1 . These two networks share the same parameters.

\section{B. Results}

The value of objective function in Equation (12) against the number of iterations for this scenario is plotted in Figure

10 The initial global cost function value of these two networks are 367,225 and 245,940 , respectively. It shows that the 
two curves decrease significantly within 10 iterations in the first step of optimization, indicating the high convergence rate of Dafermos' algorithm. The second step of optimization begins when the global cost function stops to decrease, marked by a red arrow in Figure 10 . The overall trend of the global cost function continues to decrease until the stop criterion is met. The small fluctuations are linked to the randomness of the RO algorithm. The minimum value of the global cost during the optimization process of the two networks reaches 9,068.5 and 12,963 respectively, which corresponds to a transport network with sufficiently low complexity. Compared to the initial costs, the global cost in terms of these two networks dropped nearly by $97.5 \%$ and $94.7 \%$. It can be seen that there are some plateau periods followed by sharp decreases during the second optimization step. These cases happen when the flow of a certain path becomes zero, then the complexity of the network decreases sharply.

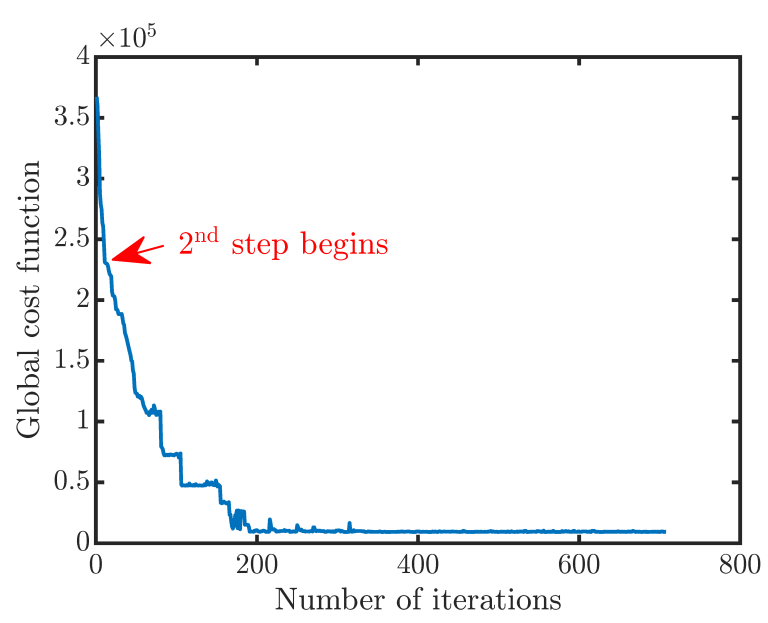

(a) transport network $G_{1}$

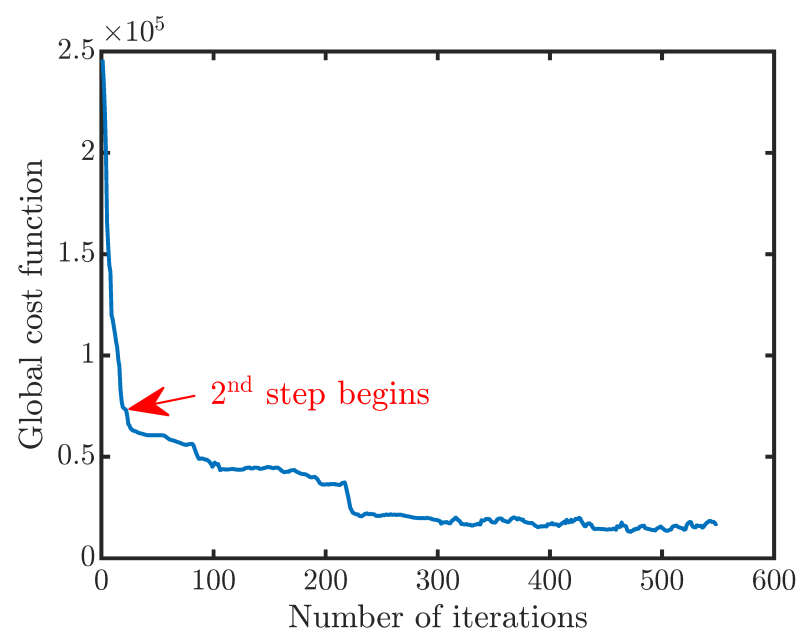

(b) transport network $G_{2}$

Fig. 10 Evolution of global cost function

The flow assigned on all paths $\left\{F_{w}^{i} \mid i \in\left[\left|1, R_{w}\right|\right]\right\}$ between OD pairs of these two networks $w \in \mathcal{W}_{1}$ and $w \in \mathcal{W}_{2}$ is illustrated in Figure 11 The paths $\left\{p_{w_{1,1}}^{1}, \ldots, p_{w_{1,1}}^{55}, p_{w_{1,2}}^{1}, \ldots, p_{w_{1,2}}^{11}\right\}$ are indexed from 1 to 66, $\left\{p_{w_{2,1}}^{1}, \ldots, p_{w_{2,1}}^{7}, p_{w_{2,2}}^{1}, \ldots, p_{w_{2,2}}^{54}\right\}$ are indexed from 1 to 61 . The final result shows that few paths are assigned with large volume of flows, and the flows on other paths are zero or close to zero. In the first network, only four paths are assigned with flows. These four paths are respectively $p_{w_{1,1}}^{6}=e_{1,1}-e_{1,4}-e_{1,9}-e_{1,6}-e_{1,21}, p_{w_{1,1}}^{9}=e_{1,1}-e_{1,4}-e_{1,10}-e_{1,12}-e_{1,21}$, $p_{w_{1,2}}^{2}=e_{1,7}-e_{1,9}-e_{1,6}-e_{1,19}$ and $p_{w_{1,2}}^{6}=e_{1,7}-e_{1,11}-e_{1,17}-e_{1,25}-e_{1,26}-e_{1,19}$. Their average length is 189.3 $\mathrm{km}$, and the average flight time is $0.96 \mathrm{~h}$. By contrast, the average length of all 66 paths are $216.2 \mathrm{~km}$, and the average flight time is $1.08 \mathrm{~h}$. In the second network, five paths are distributed with significant flow, they are $p_{w_{2,1}}^{4}=$ $e_{2,40}-e_{2,41}-e_{2,43}-e_{2,47}-e_{2,53}, p_{w_{2,2}}^{23}=e_{2,1}-e_{2,4}-e_{2,7}-e_{2,15}-e_{2,32}-e_{2,44}, p_{w_{2,2}}^{38}=e_{2,1}-e_{2,5}-e_{2,13}-e_{2,17}-e_{2,32}-e_{2,44}$, $p_{w_{2,2}}^{52}=e_{2,1}-e_{2,5}-e_{2,15}-e_{2,31}-e_{2,41}-e_{2,44}, p_{w_{2,2}}^{53}=e_{2,1}-e_{2,5}-e_{2,15}-e_{2,32}-e_{2,44}$. Their average length is 406 $\mathrm{km}$, and the average flight time is $1.88 \mathrm{~h}$. While the average length of all 61 paths are $454 \mathrm{~km}$, and the average flight time is $2.27 \mathrm{~h}$. As for both $G_{1}$ and $G_{2}$, the result indicates that these paths are efficient in terms of distance, which is able 
to reduce the flight time and delay.

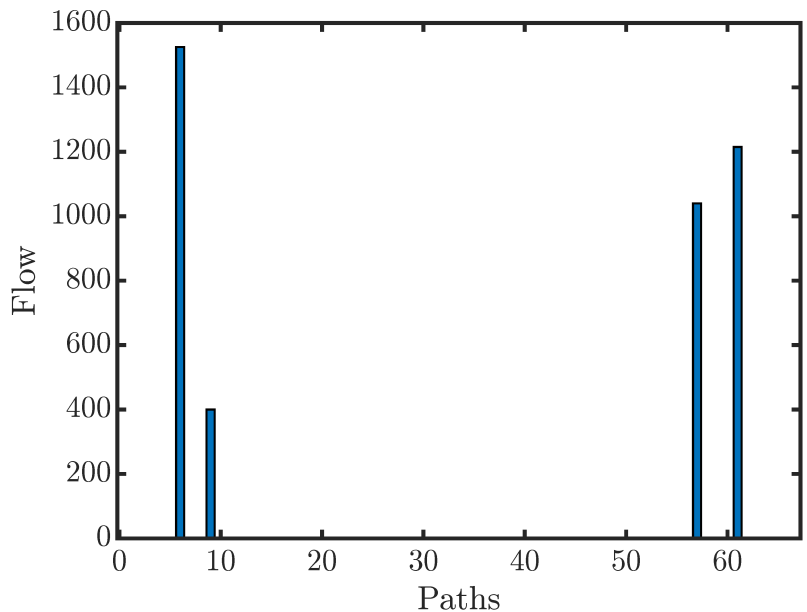

(a) transport network $G_{1}$

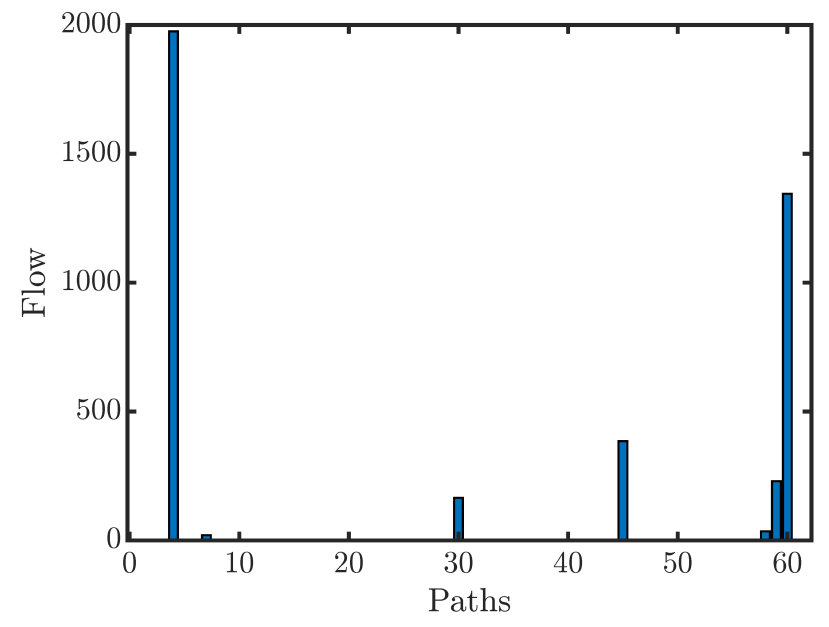

(b) transport network $G_{2}$

Fig. 11 Flow assigned on paths

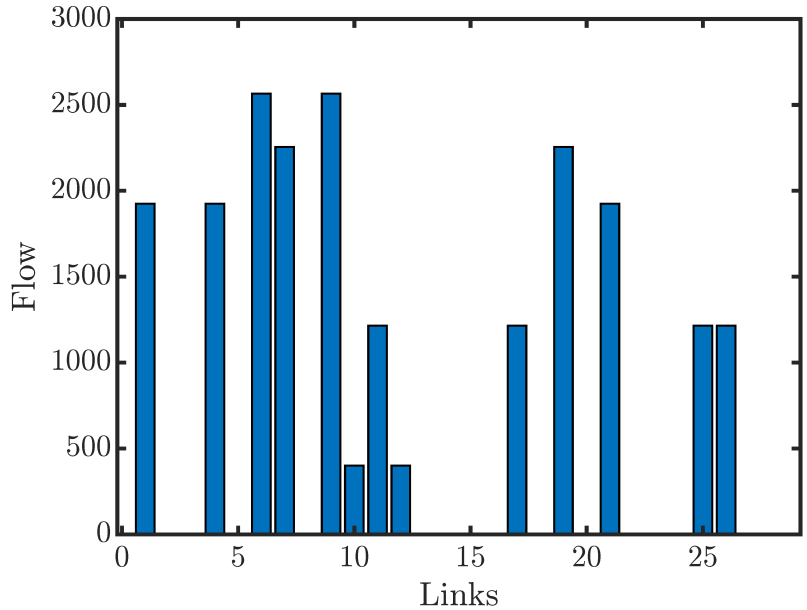

(a) transport network $G_{1}$

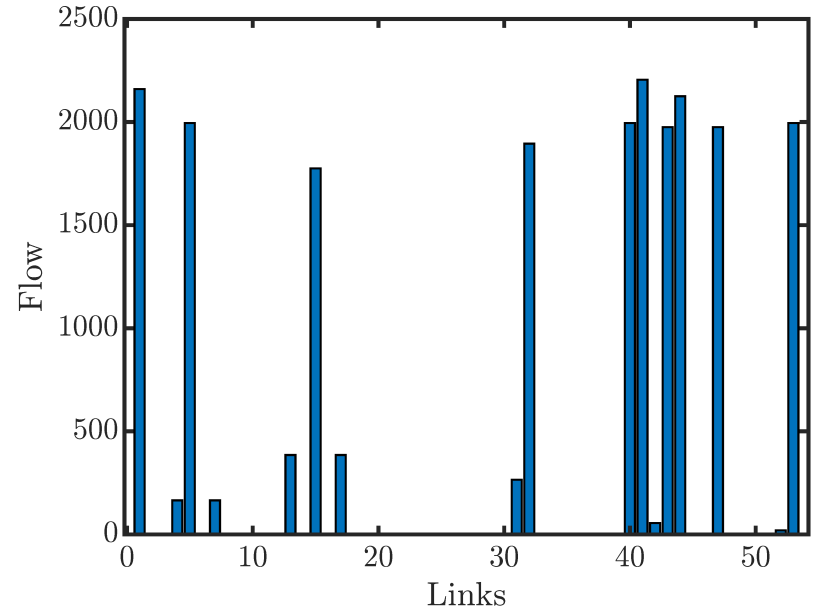

(b) transport network $G_{2}$

Fig. 12 Flow assigned on links

The flow assigned on links $\left\{f_{e} \mid e \in \mathcal{E}_{1}\right\}$ and $\left\{f_{e} \mid e \in \mathcal{E}_{2}\right\}$ are depicted in Figure 12. For the sake of a clearer visualization of the link flow and the link cost, the traffic assignment results of $G_{1}$ and $G_{2}$ in the network representation are shown in Figure 14. Figure 15 is further provided for clearer visualization, in which the links and nodes are labeled as the symbols used in the context, and only the links with nonzero flow or nonzero link cost are kept. For example, $e_{1,18}$ in Figure 14a is not allocated with flow but has nonzero link cost. The link cost is measured by different colors extracted from the colorbar, and the width of link represents the link flow. For comparison, the link costs under initial flow allocation is depicted in Figure 13 Note that, the colorbar scales in these figures are different. We can see clearly that the cost for links initially assigned with flow is extremely high, especially in dense areas. In Figure $15 \mathrm{a}$ 
in accordance with Figure $12 \mathrm{a}$, links $e_{1,1}, e_{1,4}, e_{1,6}, e_{1,7}, e_{1,9}, e_{1,10}, e_{1,11}, e_{1,12}, e_{1,17}, e_{1,19}, e_{1,21}, e_{1,25}, e_{1,26}$ have a relatively large volume of flow. From $v_{5}$ to $v_{11}$, the flows are distributed on three separate paths $: e_{9}-e_{6}, e_{10}-e_{12}$, $e_{11}-e_{17}-e_{25}-e_{26}$. By this means, the interaction between aircraft from different links is reduced to a very low level. It can be also observed that the incoming and outgoing links with similar directions are more likely to be allocated with large amount of flow, because this traffic situation complies with structuring flows, which is considered to have low traffic complexity by LDS. More specifically, $v_{1,4}, v_{1,5}, v_{1,11}$ are the nodes with high degree in $G_{1}$. In view of $v_{1,11}$, $e_{1,19}$ and $e_{1,26}$ have similar directions and are assigned with balanced flow, so that the cost for these two links are very low even if they are assigned with large amount of flow. Although the directions of $e_{1,6}$ and $e_{1,12}$ are not too different from $e_{1,21}$, the flow is distributed on link $e_{1,6}$ and $e_{1,12}$ in a way that the cost for $e_{1,21}$ is mitigated. Link $e_{1,6}$ and $e_{1,12}$ have high cost, which is unavoidable in this network with limited routes. To avoid extra traffic complexity around $v_{1,11}$, the resolution algorithm refrains to distribute the flow on $e_{1,18}$ and $e_{1,20}$. Their upstream links such as $e_{1,14}, e_{1,16}, e_{1,23}$, $e_{1,24}$ are also avoided, alleviating the complexity and congestion around node $v_{1,9}$ and $v_{1,14}$. In addition, the feasible paths containing these nodes have relatively long distances, which should also be avoided. The link configuration of node $v_{1,5}$ and $v_{1,11}$ is quite similar. The main difference is that $e_{1,6}$ and $e_{1,12}$ are incoming links of $v_{1,11}$ while $e_{1,9}$ and $e_{1,10}$ are outgoing links of $v_{1,5}$. Therefore, the costs of links $e_{1,4}, e_{1,7}$ and $e_{1,11}$ are quite high, which is in the opposite case of $e_{1,19}, e_{1,21}$ and $e_{1,26}$.

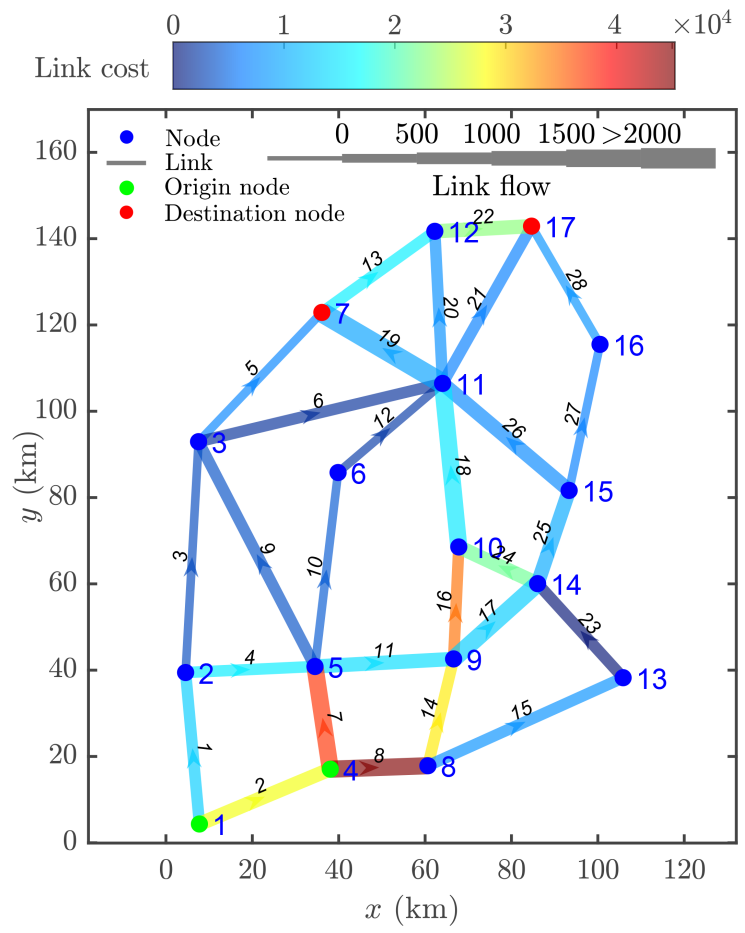

(a) transport network $G_{1}$

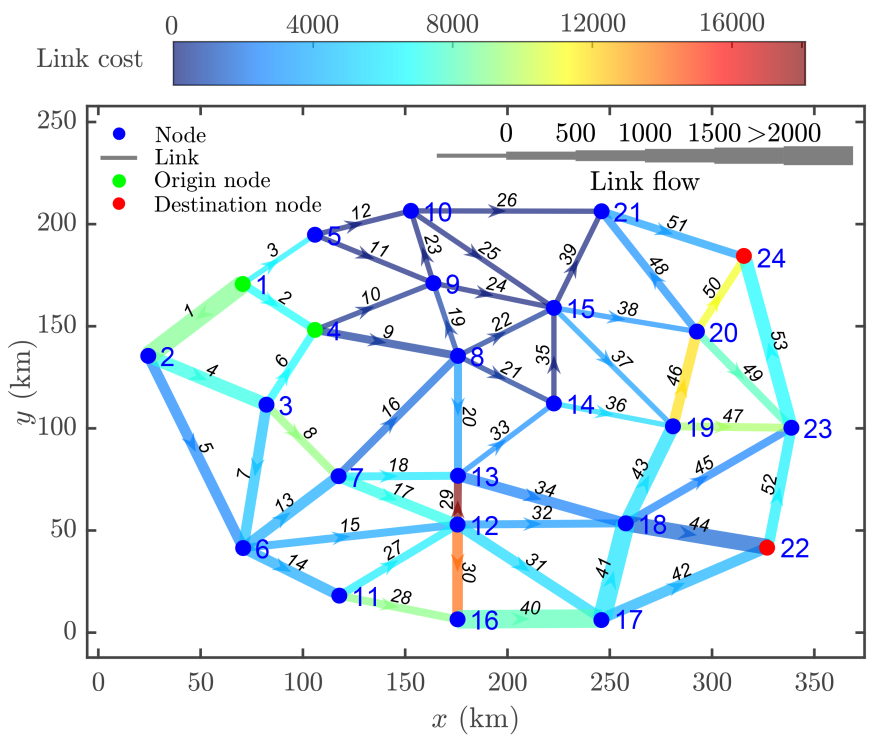

(b) transport network $G_{2}$

Fig. 13 Link costs under initial flow allocation 


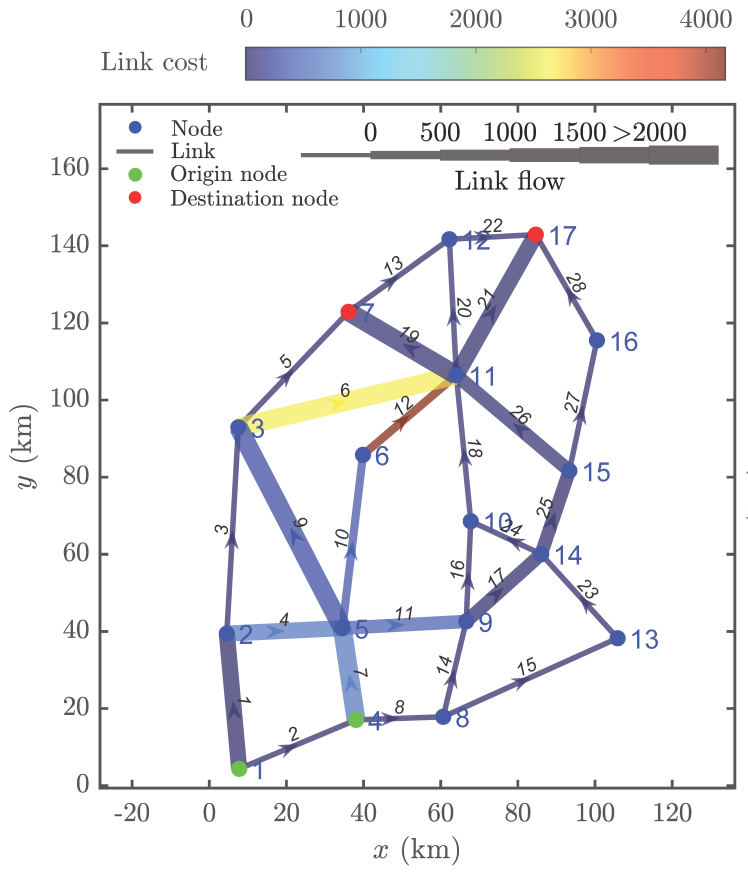

(a) transport network $G_{1}$

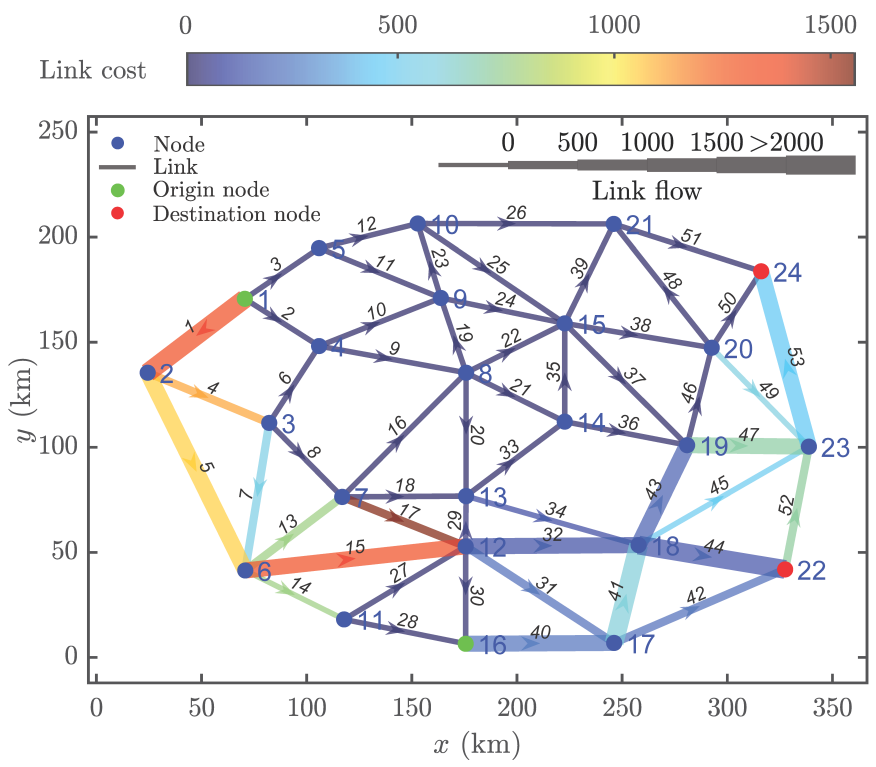

(b) transport network $G_{2}$

Fig. 14 Traffic assignment result for the whole network in terms of flow allocation and link cost

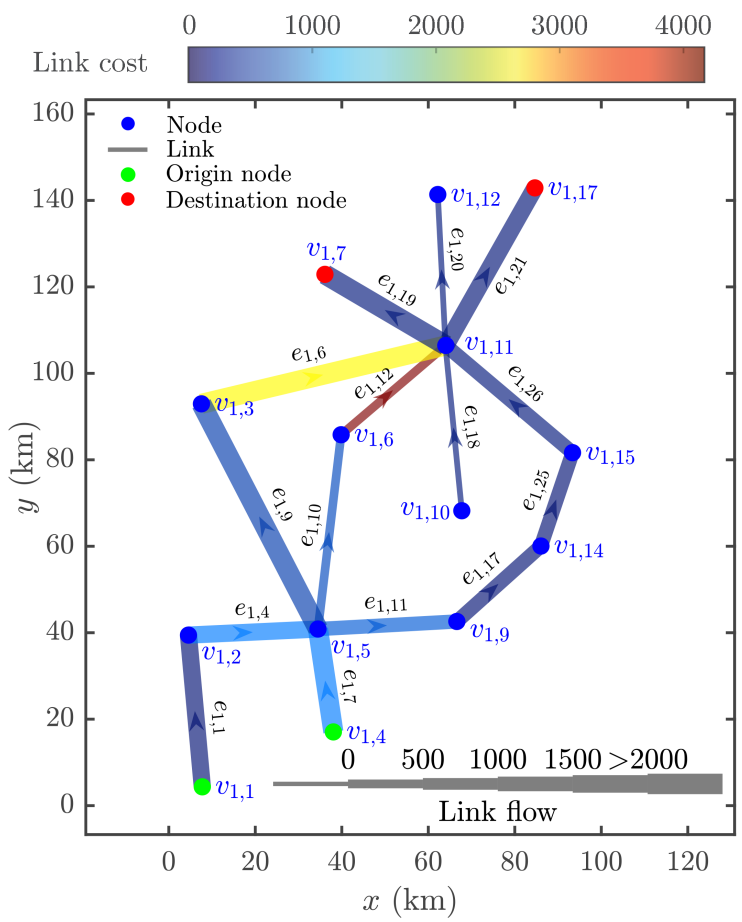

(a) transport network $G_{1}$

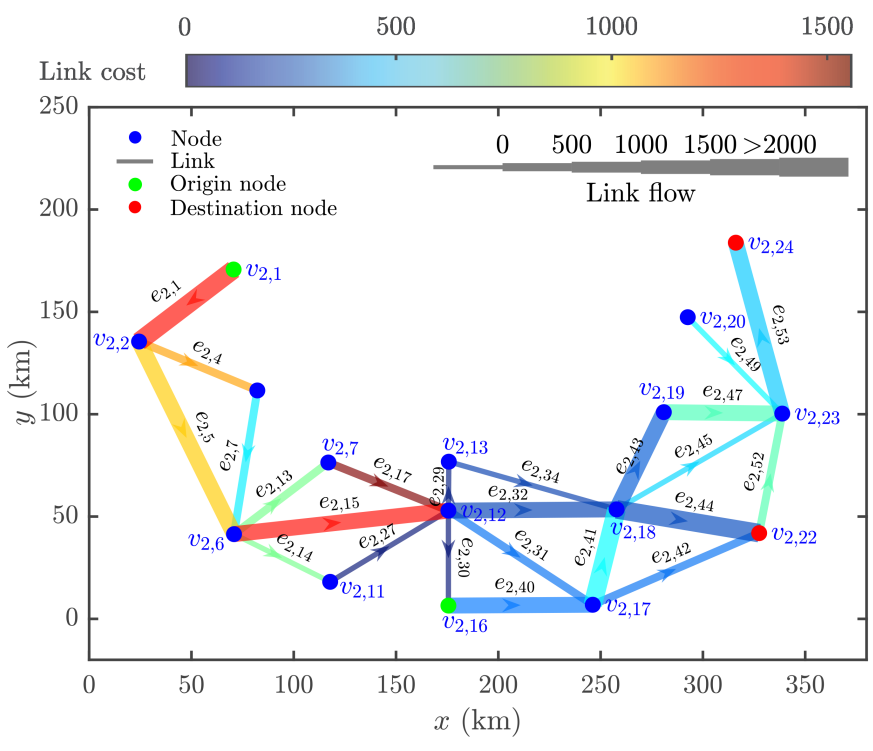

(b) transport network $G_{2}$

Fig. 15 Traffic assignment result for links with nonzero flow or nonzero link cost 
In Figure $15 \mathrm{~b}$, the network has a more complex structure. $e_{2,1}, e_{2,5}, e_{2,15}, e_{2,32}, e_{2,40}, e_{2,41}, e_{2,43}, e_{2,44}, e_{2,47}, e_{2,53}$ are allocated with massive flows, which corresponds to Figure $12 \mathrm{~b}$, It is apparent from the network representation that the upper and middle part has a complex configuration, where no flow is allocated. Especially for OD pair $w_{2,1}$, the flow is mainly distributed on path $p_{w_{2,2}}^{53}$, which is in the lower part of the network. In network $G_{2}$, the degree of nodes $v_{2,8}$, $v_{2,12}, v_{2,15}, v_{2,18}$ is relatively large and exceeds 5 . Compared with $G_{1}$, because there are more alternative feasible paths in $G_{2}$, two of these nodes $v_{2,8}$ and $v_{2,15}$ are not allocated with traffic. Besides, paths through them also have longer distances. As it can be seen from Figure 15b, links with high cost are concentrated near $v_{2,2}, v_{2,6}, v_{2,12}, v_{2,18}$ and $v_{2,23}$. The directions of links associated with $v_{2,2}$ are fairly different, so links $e_{2,1}, e_{2,4}$ and $e_{2,5}$ possess expensive costs. However, in the case that expensive link costs are inevitable, the resolution algorithm balances the link flows of each direction to reduce the cost as low as possible. $v_{2,6}$ and $v_{2,23}$ belong to almost the same case. Although $e_{2,15}$ and $e_{2,32}$ have similar directions, impacted by multiple other links related to $v_{2,6}$, link $e_{2,15}$ is quite costly. Similar illustrations happen to $e_{2,17}, e_{2,31}$ and $e_{2,41}$.

Taken together with $G_{1}$ and $G_{2}$, compared to initial flow assignment in Figure 13 their total link cost is dramatically decreased. If possible, the resolution algorithm diverts the path from complex areas. Some unnecessary or long paths may also be avoided. On the basis of the design of the link cost function, the link flow associated with the same node is balanced and optimally distributed to mitigate the congestion and traffic complexity. Even if some links are allocated with massive flows, their costs can be quite low. In addition, since the cost of the paths is the sum of the cost of included links, the paths allocated with a large amount of flow usually have short distances. With such traffic assignment results, aircraft will make effective use of networks in urban airspace. Congestion and traffic complexity are satisfying. Besides, the number and the difficulty of potential conflicts will also be strongly mitigated.

\section{Conclusion}

This paper presents a novel macroscopic air traffic assignment model to alleviate the congestion and traffic complexity of Urban Air Mobility (UAM) transport networks in future high-density urban airspace. Firstly, UAM corridors are designed which can be fitted in air transport networks in urban airspace. An intrinsic air traffic complexity metric that analyzes the airspace geometry and traffic structure is defined based on Linear Dynamical System (LDS). From a macroscopic perspective, an optimization problem under system equilibrium is formalized as an air traffic assignment model. The objective function both involves the airspace complexity metric and the congestion factors. Dafermos' is a commonly used sequential decomposition algorithm to solve this optimization problem. On that basis and in consideration of the complicated structure of the link cost function, an efficient two-step strategy is proposed to solve the associated optimization problem. To demonstrate the applicability and effectiveness of the proposed algorithm, case studies are carried out on transport networks in urban airspace. The global cost of these two networks is respectively reduced by $97.5 \%$ and $94.7 \%$ compared to the initial flow allocation. Complex areas, unnecessary and long paths can be 
avoided. The flow is balanced and optimally distributed.

In summary, this research will shed light on mitigating the traffic complexity and congestion of urban airspace based on the transport network representation from a macroscopic perspective. The proposed model is able to assist regulators and Air Navigation Service Provider (ANSP) for air traffic assignment in strategical planning of UAM operations.

Some inherent limitations which have not yet been taken into account in this work are also worth mentioning, which can be further improved in future work. More related contextual and operational constraints can be accommodated to the model, such as capacity constraints, weather conditions. Besides, other traffic flow distributions can also be explored. Last but not least, in order to have a complete view of the benefits and drawbacks of the proposed method, we plan to assess the performance of air traffic control system from various aspects, such as workload, operational and environmental metrics.

\section{Appendix}

\section{A. 2D minimum weighted mean square error estimation of linear dynamical system}

Given $N$ density points with positions $\mathbf{x}_{i}=\left(x_{i}, y_{i}\right)^{\top}$, speeds $\mathbf{v}_{i}=s_{i}\left(\cos \left(\theta_{i}\right), \sin \left(\theta_{i}\right)\right)^{\top}$, and densities $\omega_{i}, i=1, \ldots, N$, the weighted MMSE criterion between the LDS model and the observations is formalized as:

$$
E=\sum_{i=1}^{N} \omega_{i}\left\|\mathbf{v}_{i}-\left(\mathbf{A} \mathbf{x}_{i}+\mathbf{b}\right)\right\|^{2}
$$

In order to transform Equation 29] into matrix form, the following matrices are defined:

$$
\begin{gathered}
\mathbf{X}=\left[\begin{array}{cccc}
\sqrt{\omega_{1}} x_{1} & \sqrt{\omega_{2}} x_{2} & \cdots & \sqrt{\omega_{N}} x_{N} \\
\sqrt{\omega_{1}} y_{1} & \sqrt{\omega_{2}} y_{2} & \cdots & \sqrt{\omega_{N}} y_{N} \\
\sqrt{\omega_{1}} & \sqrt{\omega_{2}} & \cdots & \sqrt{\omega_{N}}
\end{array}\right] \\
\mathbf{V}=\left[\begin{array}{cccc}
\sqrt{\omega_{1}} s_{1} \cos \left(\theta_{1}\right) & \sqrt{\omega_{2}} s_{2} \cos \left(\theta_{2}\right) & \cdots & \sqrt{\omega_{N}} s_{N} \cos \left(\theta_{N}\right) \\
\sqrt{\omega_{1}} s_{1} \sin \left(\theta_{1}\right) & \sqrt{\omega_{2}} s_{2} \sin \left(\theta_{2}\right) & \cdots & \sqrt{\omega_{N}} s_{N} \sin \left(\theta_{N}\right)
\end{array}\right] \\
\mathbf{C}=\left[\begin{array}{lll}
\mathbf{A} \mid \mathbf{b}
\end{array}\right]
\end{gathered}
$$

where

$$
\mathbf{A}=\left[\begin{array}{ll}
a_{11} & a_{12} \\
a_{21} & a_{22}
\end{array}\right]
$$




$$
\mathbf{b}=\left[\begin{array}{ll}
b_{1} & b_{2}
\end{array}\right]^{\top}
$$

The error criterion $E$ can be reformalized as

$$
E=\|\mathbf{V}-\mathbf{C X}\|_{\mathrm{F}}^{2}
$$

where \|\|$_{\mathrm{F}}$ represents the Frobenius norm.

The MMSE is to find the matrix $\hat{\mathbf{C}}$ that minimize the error criterion:

$$
\hat{\mathbf{C}}=\underset{\mathbf{C} \in \mathbb{R}^{2 \times 3}}{\arg \min } E
$$

To this end, the gradient of $E$ is calculated as:

$$
\nabla_{\mathbf{C}} E=-2(\mathbf{V}-\mathbf{C X}) \mathbf{X}^{\top}
$$

$\nabla_{\mathbf{C}} E=0$ allow us to calculate $\hat{\mathbf{C}}$. If $\mathbf{X} \mathbf{X}^{\top}$ is invertible, namely the columns of $\mathbf{X}$ are linearly independent, the LDS problem has a unique solution:

$$
\hat{\mathbf{C}}=\mathbf{V} \mathbf{X}^{+}
$$

where $\mathbf{X}^{+}$is the pseudo-inverse of $\mathbf{X}$ :

$$
\mathbf{X}^{+}=\mathbf{X}^{\top}\left(\mathbf{X X}^{\top}\right)^{-1}
$$

A possible case that does not satisfy the linear independence of columns of $\mathbf{X}$ is when more than two aircraft are at the same location. This case will not happen except for collisions. Therefore, in a real traffic situation, $\mathbf{X}^{+}$always exists and is unique.

Nevertheless, LDS could be ill-conditioned in rare cases. To avoid numerical problems associated with the determinant when inversing the matrix, Singular Value Decomposition (SVD) was proposed to solve this problem. $\mathbf{X}$ can be decomposed as:

$$
\mathbf{X}=\mathbf{L} \boldsymbol{\Sigma} \mathbf{R}^{\top}
$$

where $\mathbf{L} \in \mathbb{R}^{3 \times 3}$ and $\mathbf{R} \in \mathbb{R}^{N \times N}$ are unitary matrices. $\mathbf{L}$ and $\mathbf{R}$ are not unique, which can be respectively composed by the eigenvectors of $\mathbf{X} \mathbf{X}^{\top}$ and $\mathbf{X}^{\top} \mathbf{X} . \boldsymbol{\Sigma} \in \mathbb{R}^{3 \times N}$ is a rectangular diagonal matrix with non-negative values on the diagonal.

$\mathbf{X X}^{\top} \in \mathbb{R}^{3 \times 3}$ is a symmetric matrix, and the square roots of its eigenvalues are the singular values of $\mathbf{X}$. The singular values of $\mathbf{X}$ can be sorted in descending order: $\sigma_{1} \geq \sigma_{2} \geq \cdots \geq \sigma_{r}$, where $r$ is the rank of $\mathbf{X}$ and $r \leq \min (3, N)$. Then, 
$\boldsymbol{\Sigma}$ is then defined as:

$$
\left.\boldsymbol{\Sigma}=\left[\begin{array}{cccc:ccc}
\sigma_{1} & 0 & \cdots & 0 & 0 & \cdots & 0 \\
0 & \sigma_{2} & \cdots & 0 & 0 & \cdots & 0 \\
\vdots & \vdots & \ddots & \vdots & \vdots & \ddots & \vdots \\
0 & 0 & \cdots & \sigma_{r} & 0 & \cdots & 0 \\
\hdashline 0 & 0 & \cdots & 0 & 0 & \cdots & 0 \\
\vdots & \vdots & \ddots & \vdots & \vdots & \ddots & \vdots \\
0 & 0 & \cdots & 0 & 0 & \cdots & 0
\end{array}\right]\right\} 3-r
$$

where the elements of the last $N-r$ columns and $3-r$ rows are 0 .

According to Equation 40, $\mathbf{X}^{+}$can be formalized as :

$$
\mathbf{X}^{+}=\mathbf{R} \boldsymbol{\Sigma}^{+} \mathbf{L}^{\top}
$$

where $\mathbf{R} \in \mathbb{R}^{N \times N}, \mathbf{L}^{T} \in \mathbb{R}^{3 \times 3} . \boldsymbol{\Sigma}^{+} \in \mathbb{R}^{N \times 3}$ can be formulized as:

$$
\boldsymbol{\Sigma}^{+}=\left[\begin{array}{cccc:ccc}
\frac{1}{\sigma_{1}} & 0 & \cdots & 0 & 0 & \cdots & 0 \\
0 & \frac{1}{\sigma_{2}} & \cdots & 0 & 0 & \cdots & 0 \\
\vdots & \vdots & \ddots & \vdots & \vdots & \ddots & \vdots \\
0 & 0 & \cdots & \frac{1}{\sigma_{r}} & 0 & \cdots & 0 \\
\hdashline 0 & 0 & \cdots & 0 & 0 & \cdots & 0 \\
\vdots & \vdots & \ddots & \vdots & \vdots & \ddots & \vdots \\
0 & 0 & \cdots & 0 & 0 & \cdots & 0
\end{array}\right] N-r
$$

where the elements of the last $3-r$ columns and $N-r$ rows are 0 .

$\hat{\mathbf{C}}$ is thus given by:

$$
\hat{\mathbf{C}}=\mathbf{V R} \boldsymbol{\Sigma}^{+} \mathbf{L}^{\top}
$$

Finally, $\hat{\mathbf{A}}$ can be extracted from $\hat{\mathbf{C}}$ according to Equation 32 .

\section{B. Eigenvalue loci for typical traffic situations}

Four representative examples of traffic situations and the associated location of their eigenvalues in the complex plane are illustrated in Figure 16 [44]. These cases include pure translation, convergence, divergence, and pure rotation. 
As we can see, the eigenvalues of pure translation and pure rotation are located in the strip represented in Figure 5 , so they correspond to organized traffic situations. In the translation case, the eigenvalues are zero, the speed vectors are parallel and their relative distances remain the same. In the convergence case, the eigenvalues are negative real, and the LDS is in contraction mode. Their relative distances reduce with time and the points are converging. In the divergence case, the eigenvalues are positive real and the LDS is in expansion mode. The aircraft are diverging and their relative distances increase with time. The second and the third case belong to unorganized traffic patterns because the relative distance between aircraft varies with time. For the rotation case, the eigenvalues are imaginary. Although the points move around a circle, their relative distance is constant.

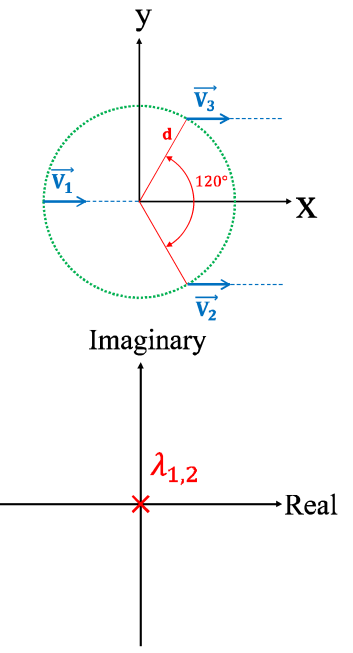

(a) Translation
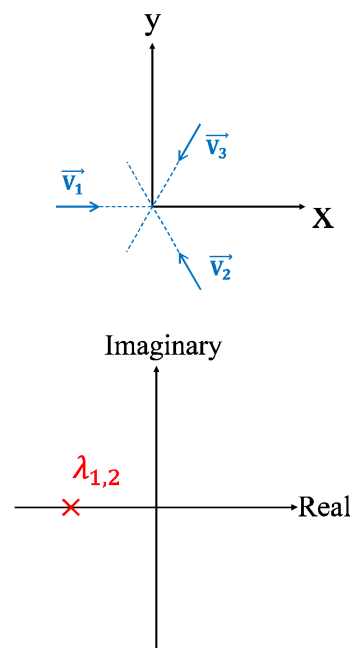

(b) Convergence
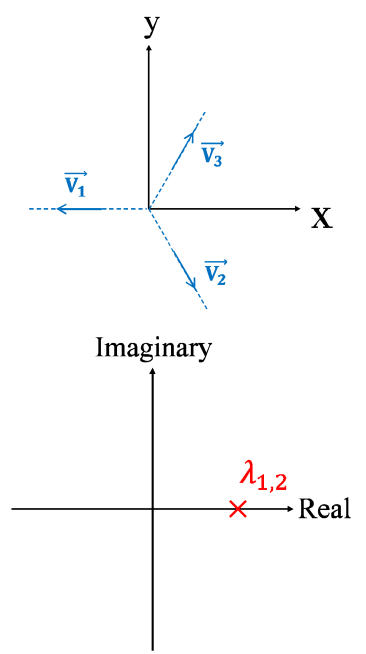

(c) Divergence
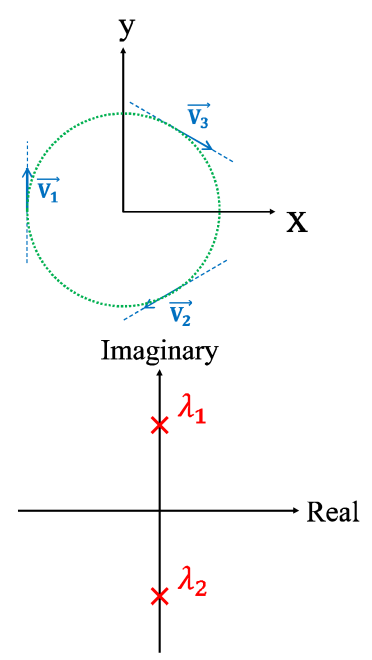

(d) Rotation

Fig. 16 Eigenvalue loci for four typical traffic situations

\section{Funding Sources}

This work is partially supported by the research project CONCORDE of the Defense Innovation Agency (AID) of the French Ministry of Defense (2019650090004707501).

\section{References}

[1] Vascik, P. D., and Hansman, R. J., "Scaling Constraints for Urban Air Mobility Operations: Air Traffic Control, Ground Infrastructure, and Noise," 2018 Aviation Technology, Integration, and Operations Conference, American Institute of Aeronautics and Astronautics, 2018, p. 3849. https://doi.org/10.2514/6.2018-3849

[2] Thipphavong, D. P., Apaza, R., Barmore, B., Battiste, V., Burian, B., Dao, Q., Feary, M., Go, S., Goodrich, K. H., Homola, J., et al., "Urban air mobility airspace integration concepts and considerations," 2018 Aviation Technology, Integration, and Operations Conference, 2018, p. 3676. 
[3] Schneider, O., Kern, S., Knabe, F., Gerdes, I., Delahaye, D., Vidosavljevic, A., van Leeuwen, P., Nieuwenhuisen, D., Sunil, E., Hoekstra, J., and Ellerbroek, J., “Metropolis - Urban Airspace Design,” Tech. rep., The European Commission, 2015.

[4] Administration, F. A., "Urban Air Mobility (UAM) concepts of operations v1.0," Tech. rep., U.S. department of transportation, 2020.

[5] Singireddy, S. R. R., and Daim, T. U., “Technology Roadmap: Drone Delivery - Amazon Prime Air,” Innovation, Technology, and Knowledge Management, Springer International Publishing, 2018, pp. 387-412. https://doi.org/10.1007/978-3-319-68987-6_13

[6] Salleh, M. F. B. M., Wanchao, C., Wang, Z., Huang, S., Tan, D. Y., Huang, T., and Low, K. H., "Preliminary Concept of Adaptive Urban Airspace Management for Unmanned Aircraft Operations," 2018 AIAA Information Systems-AIAA Infotech @ Aerospace, American Institute of Aeronautics and Astronautics, 2018, p. 2260. https://doi.org/10.2514/6.2018-2260

[7] Hoekstra, J., Kern, S., Schneider, O., Knabe, F., and Lamiscarre, B., “Metropolis Scenario Definition Report,” Tech. rep., TU Delft, 2015.

[8] Ma, C., Cai, Q., Alam, S., Sridhar, B., and Duong, V. N., “Airway network management using Braess's Paradox,” Transportation Research Part C: Emerging Technologies, Vol. 105, No. February, 2019, pp. 565-579. https://doi.org/10.1016/j.trc.2019.06.014

[9] David, J. W., and Ian, M. L., "New Flight Rules to Enable the Era of Aerial Mobility in the National Airspace System," Tech. Rep. 20205008308, NASA Langley Research Center, VA, United States, November 2020.

[10] Bruno, G., Esposito, E., and Genovese, A., "A model for aircraft evaluation to support strategic decisions," Expert Systems with Applications, Vol. 42, No. 13, 2015, pp. 5580-5590. https://doi.org/10.1016/j.eswa.2015.02.054

[11] Patriksson, M., The traffic assignment problem: models and methods, Courier Dover Publications, 2015.

[12] Smock, R. B., “A comparative description of a capacity-restrained traffic assignment," Highway Research Record, 1963.

[13] Mosher Jr, W. W., “A capacity-restraint algorithm for assigning flow to a transport network," Highway Research Record, 1963.

[14] Spiess, H., “Technical Note-Conical Volume-Delay Functions,” Transportation Science, Vol. 24, No. 2, 1990 , pp. $153-158$. https://doi.org/10.1287/trsc.24.2.153

[15] Huntsinger, L. F., and Rouphail, N. M., "Bottleneck and Queuing Analysis," Transportation Research Record: Journal of the Transportation Research Board, Vol. 2255, No. 1, 2011, pp. 117-124. https://doi.org/10.3141/2255-13

[16] Neuhold, R., and Fellendorf, M., "Volume Delay Functions Based on Stochastic Capacity," Transportation Research Record: Journal of the Transportation Research Board, Vol. 2421, No. 1, 2014, pp. 93-102. https://doi.org/10.3141/2421-11

[17] Wang, T.-G., Xie, C., Xie, J., and Waller, T., "Path-constrained traffic assignment: A trip chain analysis under range anxiety," Transportation Research Part C: Emerging Technologies, Vol. 68, 2016, pp. 447-461. https://doi.org/10.1016/j.trc.2016.05.003

[18] Ryu, S., Chen, A., Su, J., and Choi, K., “Two-Stage Bicycle Traffic Assignment Model,” Journal of Transportation Engineering, Part A: Systems, Vol. 144, No. 2, 2018, p. 04017079. https://doi.org/10.1061/jtepbs.0000108 
[19] Patil, G. R., "Emission-based static traffic assignment models," Environmental Modeling \& Assessment, Vol. 21, No. 5, 2015, pp. 629-642. https://doi.org/10.1007/s10666-015-9498-7

[20] Tidswell, J., and Raith, A., "Modelling traffic assignment objectives with emission cost functions," Australasian Transport Research Forum (ATRF), 39th, 2017, Auckland, New Zealand, Vol. 495, 2017.

[21] Lin, H.-Z., and Wei, J., "Optimal transport network design for both traffic safety and risk equity considerations," Journal of Cleaner Production, Vol. 218, 2019, pp. 738-745. https://doi.org/10.1016/j.jclepro.2019.02.070

[22] Yang, H., and Yagar, S., "Traffic assignment and signal control in saturated road networks," Transportation Research Part A: Policy and Practice, Vol. 29, No. 2, 1995, pp. 125-139. https://doi.org/10.1016/0965-8564(94)e0007-v

[23] Kamel, I., Shalaby, A., and Abdulhai, B., "Integrated simulation-based dynamic traffic and transit assignment model for large-scale network," Canadian Journal of Civil Engineering, Vol. 47, No. 8, 2020, pp. 898-907. https://doi.org/10.1139/cjce-2018-0706

[24] Treimuth, T., Delahaye, D., Puechmorel, S., and Ngueveu, S. U., "Parallel complexity computation based on dynamical systems," 2015 IEEE/AIAA 34th Digital Avionics Systems Conference (DASC), IEEE, 2015, pp. 1C2-1. https://doi.org/10.1109/dasc.2015. 7311340

[25] Delahaye, D., and Puechmorel, S., "Air traffic complexity: towards intrinsic metrics," Proceedings of the third USA/Europe Air Traffic Management R \& D Seminar, Vol. 505, 2000.

[26] Lee, K., Feron, E., and Pritchett, A., "Air Traffic Complexity: An Input-Output Approach,” 2007 American Control Conference, IEEE, 2007, pp. 474-479. https://doi.org/10.1109/acc.2007.4282989

[27] Davis, C., Danaher, J., and Fischl, M., "The influence of selected sector characteristics upon ARTCC controller activities," Contract No. FAA/BRD-301). Arlington, VA: Matrix Corporation, 1963.

[28] Arad, B. A., “The control load and sector design,” Journal of Air Traffic Control, 1964.

[29] Meckiff, C., Chone, R., and Nicolaon, J.-P., “The tactical load smoother for multi-sector planning," Proceedings of the 2nd usa/europe air traffic management research and development seminar, 1998, pp. 1-12.

[30] David K. Schmidt, “A queueing analysis on the air traffic controller's workload,” IEEE Transactions on Systems, Man, and Cybernetics, Vol. 8, 1978, pp. 492-498.

[31] Janić, M., and Tošić, V., “En route sector capacity model,” Transportation Science, Vol. 25, No. 4, 1991, pp. $299-307$.

[32] Maugis, L., and Gotteland, J. B., “Techniques de détermination de la capacité des secteurs de contrôle de l'espace aérien: statistiques et simulations," Centre d'études de la navigation aérienne, France, 1997.

[33] Delahaye, D., and Odoni, A. R., "Airspace congestion smoothing by stochastic optimization," Evolutionary Programming VI, Springer Berlin Heidelberg, 1997, pp. 163-176. https://doi.org/10.1007/bfb0014809 
[34] Laudeman, I. V., Shelden, S. G., Branstrom, R., and Brasil, C. L., "Dynamic density: An air traffic management metric (NASA/TM-1998-112226)," Tech. rep., NASA, 1998.

[35] Sridhar, B., Sheth, K. S., and Grabbe, S., "Airspace complexity and its application in air traffic management," 2nd USA/Europe Air Traffic Management R\&D Seminar, 1998, pp. 1-6.

[36] Lee, K., Feron, E., and Pritchett, A., “Describing Airspace Complexity: Airspace Response to Disturbances,” Journal of Guidance, Control, and Dynamics, Vol. 32, No. 1, 2009, pp. 210-222. https://doi.org/10.2514/1.36308

[37] Granger, G., and Durand, N., “A traffic complexity approach through cluster analysis," Proceedings of the 5th ATM R\&D Seminar, Budapest, 2003.

[38] Delahaye, D., Vidosavljevic, A., Sunil, E., Hoekstra, J., Ellerbroek, J., and Aalmoes, R., "Metropolis - Development \& Metrics Definition,” Tech. rep., ENAC, 2015.

[39] Histon, J. M., Hansman, R. J., Aigoin, G., Delahaye, D., and Puechmorel, S., "Introducing Structural Considerations into Complexity Metrics," Air Traffic Control Quarterly, Vol. 10, No. 2, 2002, pp. 115-130. https://doi.org/10.2514/atcq.10.2.115

[40] Delahaye, D., and Puechmorel, S., "Modeling and Optimization of Air Traffic," Modeling and Optimization of Air Traffic, 2013, pp. 1-329. https://doi.org/10.1002/9781118743805

[41] Essén, H., “Average angular velocity,” European Journal of Physics, Vol. 14, No. 5, 1993, pp. 201-205. https://doi.org/10.1088/ $0143-0807 / 14 / 5 / 002$

[42] Delahaye, D., Paimblanc, P., Puechmorel, S., Histon, J., and Hansman, R., "A new air traffic complexity metric based on dynamical system modelization," Proceedings. The 21st Digital Avionics Systems Conference, Vol. 1, IEEE, 2002 , pp. 4A2-4A2. https://doi.org/10.1109/dasc.2002.1067960

[43] Juntama, P., Chaimatanan, S., Alam, S., and Delahaye, D., “A Distributed Metaheuristic Approach for Complexity Reduction in Air Traffic for Strategic 4D Trajectory Optimization,” 2020 International Conference on Artificial Intelligence and Data Analytics for Air Transportation (AIDA-AT), IEEE, 2020, pp. 1-9. https://doi.org/10.1109/aida-at48540.2020.9049200.

[44] Delahaye, D., Puechmorel, S., Hansman, J., and Histon, J., “Air Traffic Complexity Map based on Non Linear Dynamical Systems," Air Traffic Control Quarterly, Vol. 12, No. 4, 2004, pp. 367-388. https://doi.org/10.2514/atcq.12.4.367

[45] Delahaye, D., and Puechmorel, S., "Air traffic complexity based on dynamical systems," 49th IEEE Conference on Decision and Control (CDC), IEEE, 2010. https://doi.org/10.1109/cdc.2010.5718004

[46] Delahaye, D., Puechmorel, S., Hansman, J., and Histon, J., "Air Traffic Complexity Map based on Non Linear Dynamical Systems," Air Traffic Control Quarterly, Vol. 12, No. 4, 2004, pp. 367-388. https://doi.org/10.2514/atcq.12.4.367

[47] García, A., Delahaye, D., and Soler, M., “Air Traffic Complexity Map based on Linear Dynamical Systems,”, 2020. Working paper or preprint. 
[48] Barnhart, C., Fearing, D., Odoni, A., and Vaze, V., "Demand and capacity management in air transportation," EURO Journal on Transportation and Logistics, Vol. 1, No. 1-2, 2012, pp. 135-155. https://doi.org/10.1007/s13676-012-0006-9

[49] Chaimatanan, S., Delahaye, D., and Mongeau, M., "A Hybrid Metaheuristic Optimization Algorithm for Strategic Planning of 4D Aircraft Trajectories at the Continental Scale," IEEE Computational Intelligence Magazine, Vol. 9, No. 4, 2014 , pp. 46-61. https://doi.org/10.1109/mci.2014.2350951

[50] Delahaye, D., and Puechmorel, S., Modeling and Optimization of Air Traffic, Wiley Online Library, 2013. https://doi.org/10. $1002 / 9781118743805$

[51] Ferguson, A. R., and Dantzig, G. B., “The problem of routing aircraft: A mathematical solution,” Tech. rep., RAND PROJECT AIR FORCE ARLINGTON VA, 1954.

[52] Farges, J.-L., and Delahaye, D., "Pricing Policies for Air Traffic Assignment," Air Transportation Systems Engineering, 2001, pp. 143-157. https://doi.org/10.2514/5.9781600866630.0143.0157.

[53] Deschinkel, K., Farges, J.-L., and Delahaye, D., "Optimizing and assigning price levels for air traffic management,” Transportation Research Part E: Logistics and Transportation Review, Vol. 38, No. 3-4, 2002, pp. 221-237. https://doi.org/10.1016/s1366$5545(02) 00007-8$

[54] Daniel, D., Oussedik, S., and Stephane, P., "Airspace congestion smoothing by multi-objective genetic algorithm," Proceedings of the 2005 ACM symposium on Applied computing - SAC '05, ACM Press, 2005, pp. 907-912. https://doi.org/10.1145/1066677. 1066887

[55] Nosedal, J., Piera, M. A., Ruiz, S., and Nosedal, A., "An efficient algorithm for smoothing airspace congestion by finetuning take-off times," Transportation Research Part C: Emerging Technologies, Vol. 44, 2014, pp. 171-184. https: //doi.org/10.1016/j.trc.2014.03.017.

[56] Strub, I., and Bayen, A., "Optimal Control of Air Traffic Networks Using Continuous Flow Models," AIAA Guidance, Navigation, and Control Conference and Exhibit, American Institute of Aeronautics and Astronautics, 2006, p. 6228. https://doi.org/10.2514/6.2006-6228

[57] Bertsimas, D., Lulli, G., and Odoni, A., "An Integer Optimization Approach to Large-Scale Air Traffic Flow Management," Operations Research, Vol. 59, No. 1, 2011, pp. 211-227. https://doi.org/10.1287/opre.1100.0899.

[58] Zhang, M., quan Cai, K., and bo Zhu, Y., "An improved multi-objective particle swarm optimizer for air traffic flow network rerouting problem," 2012 IEEE/AIAA 31st Digital Avionics Systems Conference (DASC), IEEE, 2012, pp. 4B4-1. https://doi.org/10.1109/dasc.2012.6382335

[59] Zhang, X., Guan, X., Zhu, Y., and Lei, J., "Strategic flight assignment approach based on multi-objective parallel evolution algorithm with dynamic migration interval," Chinese Journal of Aeronautics, Vol. 28, No. 2, 2015, pp. 556-563. https://doi.org/10.1016/j.cja.2015.01.012 
[60] Delahaye, D., and Puechmorel, S., "Genetic Algorithms and Improvements," Modeling and Optimization of Air Traffic, John Wiley \& Sons, Inc., 2013, pp. 37-66. https://doi.org/10.1002/9781118743805.ch2

[61] Haouari, M., Aissaoui, N., and Mansour, F. Z., "Network flow-based approaches for integrated aircraft fleeting and routing," European Journal of Operational Research, Vol. 193, No. 2, 2009, pp. 591-599. https://doi.org/10.1016/j.ejor.2007.11.042.

[62] Jiang, X., Zhou, Q., and Ye, Y., "Method of Task Assignment for UAV Based on Particle Swarm Optimization in logistics," Proceedings of the 2017 International Conference on Intelligent Systems, Metaheuristics \& Swarm Intelligence - ISMSI '17, ACM Press, 2017, pp. 113-117. https://doi.org/10.1145/3059336.3059337

[63] Zhou, Z., Feng, J., Gu, B., Ai, B., Mumtaz, S., Rodriguez, J., and Guizani, M., "When Mobile Crowd Sensing Meets UAV: Energy-Efficient Task Assignment and Route Planning," IEEE Transactions on Communications, Vol. 66, No. 11, 2018, pp. 5526-5538. https://doi.org/10.1109/tcomm.2018.2857461.

[64] Cheng, L., Zhong, L., Tian, S., and Xing, J., “Task Assignment Algorithm for Road Patrol by Multiple UAVs With Multiple Bases and Rechargeable Endurance,” IEEE Access, Vol. 7, 2019, pp. 144381-144397. https://doi.org/10.1109/access.2019.2944881.

[65] Liu, D., Wang, J., Xu, K., Xu, Y., Yang, Y., Xu, Y., Wu, Q., and Anpalagan, A., “Task-Driven Relay Assignment in Distributed UAV Communication Networks," IEEE Transactions on Vehicular Technology, Vol. 68, No. 11, 2019, pp. $11003-11017$. https://doi.org/10.1109/tvt.2019.2942095.

[66] Netjasov, F. T., "A model of air traffic assignment as a measure for mitigating noise at airports: The Zurich Airport Case," Transportation Planning and Technology, Vol. 31, No. 5, 2008, pp. 487-508. https://doi.org/10.1080/03081060802364448

[67] Ganić, E., Babić, O., Čangalović, M., and Stanojević, M., "Air traffic assignment to reduce population noise exposure using activity-based approach," Transportation Research Part D: Transport and Environment, Vol. 63, 2018 , pp. 58-71. https://doi.org/10.1016/j.trd.2018.04.012.

[68] Chatelain, P., and Van Vyve, M., "Modeling fair air traffic assignment in the vicinity of airports," Transportation Research Part D: Transport and Environment, Vol. 65, 2018, pp. 213-228. https://doi.org/10.1016/j.trd.2018.08.016

[69] Ho-Huu, V., Ganić, E., Hartjes, S., Babić, O., and Curran, R., "Air traffic assignment based on daily population mobility to reduce aircraft noise effects and fuel consumption," Transportation Research Part D: Transport and Environment, Vol. 72, 2019, pp. 127-147. https://doi.org/10.1016/j.trd.2019.04.007

[70] Economou, J. T., Kladis, G., Tsourdos, A., and White, B. A., "UAV optimum energy assignment using Dijkstra's Algorithm,” 2007 European Control Conference (ECC), 2007, pp. 287-292. https://doi.org/10.23919/ECC.2007.7068353.

[71] Mirosavljević, P., Gvozdenović, S., and Čokorilo, O., "A model of air traffic assignment as part of airport air pollution management system,” Aviation, Vol. 15, No. 4, 2011, pp. 92-100. https://doi.org/10.3846/16487788.2011.651792

[72] Vascik, P. D., Hansman, R. J., and Dunn, N. S., "Analysis of Urban Air Mobility Operational Constraints," Journal of Air Transportation, Vol. 26, No. 4, 2018, pp. 133-146. https://doi.org/10.2514/1.d0120 
[73] Migliore, M., Martorana, V., and Sciortino, F., "An algorithm to find all paths between two nodes in a graph," Journal of Computational Physics, Vol. 87, No. 1, 1990, pp. 231-236. https://doi.org/10.1016/0021-9991(90)90235-s

[74] Johnson, W., "UAM Coordination and Assessment Team (UCAT) NASA UAM for ENRI Technical Interchange Meeting,” Tech. Rep. 20190025373, NASA Langley Research Center, VA, United States, May 2019.

[75] WARDROP, J. G., "ROAD PAPER. SOME THEORETICAL ASPECTS OF ROAD TRAFFIC RESEARCH.” Proceedings of the Institution of Civil Engineers, Vol. 1, No. 3, 1952, pp. 325-362. https://doi.org/10.1680/ipeds.1952.11259

[76] Dafermos, S. C., and Sparrow, F. T., "The traffic assignment problem for a general network," Journal of Research of the National Bureau of Standards B, Vol. 73, No. 2, 1969, pp. 91-118.

[77] Dafermos, S. C., “An Extended Traffic Assignment Model with Applications to Two-Way Traffic," Transportation Science, Vol. 5, No. 4, 1971, pp. 366-389. https://doi.org/10.1287/trsc.5.4.366

[78] Dafermos, S. C., “The Traffic Assignment Problem for Multiclass-User Transportation Networks,” Transportation Science, Vol. 6, No. 1, 1972, pp. 73-87. https://doi.org/10.1287/trsc.6.1.73.

[79] Ewedairo, K., Chhetri, P., and Jie, F., "Estimating transportation network impedance to last-mile delivery," The International Journal of Logistics Management, Vol. 29, No. 1, 2018, pp. 110-130. https://doi.org/10.1108/ijlm-10-2016-0247 